\title{
A SGAM-Based Test Platform to Develop a Scheme for Wide Area Measurement-Free Monitoring of Smart Grids under High PV Penetration
}

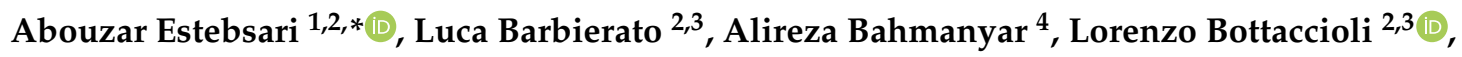 \\ Enrico Macii ${ }^{5}$ and Edoardo Patti ${ }^{2,3}$ (D) \\ 1 Department of Energy, Politecnico di Torino, 10129 Torino, Italy \\ 2 Energy Center Lab, Politecnico di Torino, 10129 Torino, Italy; luca.barbierato@polito.it (L.B.); \\ lorenzo.bottaccioli@polito.it (L.B.); edoardo.patti@polito.it (E.P.) \\ 3 Department of Control and Computer Engineering, Politecnico di Torino, 10129 Torino, Italy \\ 4 Department of Electrical Engineering, Iran University of Science and Technology, Tehran 16846-13114, Iran; \\ bahmanyar@alumni.iust.ac.ir \\ 5 Interuniversity Department of Regional and Urban Studies and Planning, Politecnico di Torino, \\ 10129 Torino, Italy; enrico.macii@polito.it \\ * Correspondence: abouzar.estebsari@polito.it
}

Received: 20 February 2019; Accepted: 3 April 2019; Published: 12 April 2019

\begin{abstract}
In order to systematically shift existing control and management paradigms in distribution systems to new interoperable communication supported schemes in smart grids, we need to map newly developed use cases to standard reference models like Smart Grid Architecture Model (SGAM). From the other side, any new use cases should be tested and validated ex-ante before being deployed in the real-world system. Considering various types of actors in smart grids, use cases are usually tested using co-simulation platforms. Currently, there is no efficient co-simulation platform which supports interoperability analysis based on SGAM. In this paper, we present our developed test platform which offers a support to design new use cases based on SGAM. We used this platform to develop a new scheme for wide area monitoring of existing distribution systems under growing penetration of Photovoltaic production. Off-the-shelf solutions of state estimation for wide area monitoring are either used for passive distribution grids or applied to the active networks with wide measurement of distributed generators. Our proposed distribution state estimation algorithm does not require wide area measurements and relies on the data provided by a PV simulator we developed. This practical scheme is tested experimentally on a realistic urban distribution grid. The monitoring results shows a very low error rate of about $1 \%$ by using our PV simulator under high penetration of PV with about $30 \%$ error of load forecast. Using our SGAM-based platform, we could propose and examine an Internet-of-Things-based infrastructure to deploy the use case.
\end{abstract}

Keywords: smart grid architecture model; state estimation; wide area monitoring; distribution systems; real time simulation; PV penetration

\section{Introduction}

Nowadays, we are moving from hierarchical power distribution networks to smart distributed systems, where control policies (or services in general) and Renewable Energy Sources (RES) are pervasively deployed across distribution networks. This distributed power network, also known as smart grid, must provide features to be self-healing and resilient to anomalies [1]. To foster this transition and to systematically shift existing control and protection paradigms to a full smart grid view, we need to map new use cases and services to Smart Grid Architecture Model (SGAM) [2]. 
As defined in [2], SGAM is a methodology that helps in designing new smart grid's use cases and services with an architectural approach. This methodology allows a neutral representation of the involved technologies highlighting their interoperability supported by standards and, consequently, enabling standards gap analysis. For this purpose, SGAM defines a three-dimensional model (see Figure 1) consisting of five interoperable layers: (i) Component Layer, (ii) Communication Layer, (iii) Information Layer, (iv) Function Layer and (v) Business Layer.

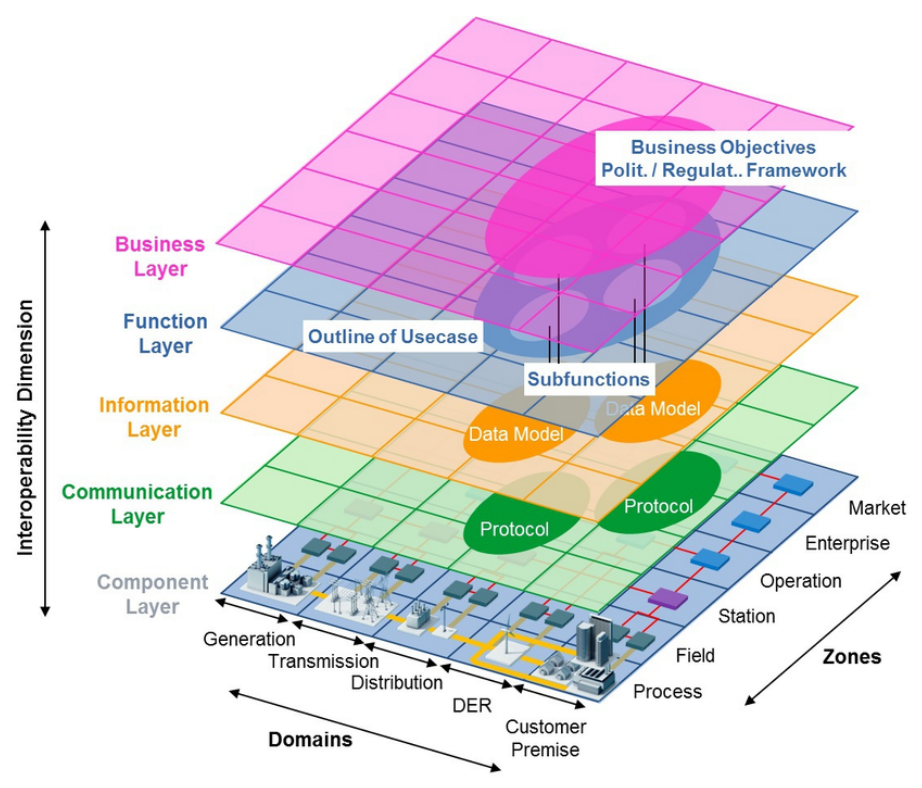

Figure 1. Smart grid architecture model (SGAM) with interoperability layers.

To test and validate novel services and algorithms for smart grids, a near real-world environment is needed [1]. Hence, to study the interoperability in smart grids based on SGAM, we need to combine different tools and simulators that concurrently reproduce the behaviour of the five SGAM layers for different use cases. Thus, we need a distributed real-time co-simulation framework able to simulate systems and to test and verify performance of new general-purpose services. In this view, distributed real-time simulations (RTS) can support the research efforts to meet emerging lab test requirements [3-5].

RTS is the most reliable tool for electromagnetic transient analysis (EMT) in power systems, which is greatly flexible and scalable thanks to its computation parallelism feature. Different software algorithms and functions, as well as various hardware devices, could be tested using RTS through so-called software in-the-loop and hardware in-the-loop set-ups. This can reduce costs and enable more complete and continuous testing of the entire system without interruption, with many possible configurations, and safely under possibly dangerous conditions.

Considering the importance of both SGAM and co-simulation, and noting the great contribution which RTS could add, we developed a distributed SGAM-based co-simulation platform that integrates RTS functionalities with distributed software components for smart grid management (e.g., device management, data collection and services) so that use case developers could also partially use real-world systems by coupling hardware devices or using actual measurements in real-time. The system under test could be replaced by the real physical system as the last phase of test and validation before completely deploying the new system in the field. In this view, all physical components of the electric system can be modelled and simulated by Digital Real Time Simulators (DRTSs). However, some of these components like intelligent electronic devices including meters could be decoupled from the core of DRTS, in case they are real (physical) devices or prototypes. This so-called hardware in-the-loop experiment enables more complete and real-like testing of the system for interoperability analysis of new devices. 
ICT (Information and Communication Technology) infrastructure gives to the Smart Grid the character of a complex cyber-physical system [6]: hardware and software elements acting together to foster innovative automation and control process in the grid management. Unfortunately, the cost of developing and deploying real-world testbed to assess innovation feasibility is too high and risky. Resiliency of the power grid could come under stress during the testing of new Smart Grid technologies. Hence, the co-simulation of the communication network and the power system infrastructure has become of primary importance in the Smart Grid challenge to determine the benefit of these innovation $[7,8]$.

In this paper, we present a novel distributed real-time co-simulation framework based on SGAM. To prove platform functionalities, we also propose a novel algorithm for wide area measurement-free monitoring of smart grids under high PV Penetration. Our co-simulation framework is flexible on integrating and testing different services, even concurrent, to realize distributed intelligence in smart grids. The scientific novelty of the proposed co-simulation framework consists of:

- $\quad$ providing a test and validation tool to evaluate performance and operation of novel smart grid's use cases based on SGAM;

- $\quad$ providing modularity, flexibility and scalability to easily test new use cases in a plug-and-play fashion;

- allowing an interoperability analysis of all the entities either hardware or software) involved in the simulation scenario;

- integrating digital real-time simulators to grant even the opportunity of performing hardware in-the-loop simulations;

- integrating ICT network simulators (i.e., OMNeT++ [9]) to study the impact of new Internet-connected technologies (i.e., Internet-of-Things devices) on the communication networks;

- integrating third party software (e.g., smart metering architectures [10]) to allow distributed software in-the-loop simulations by exploiting even data collected by real-world Internet-of-Things (IoT) devices.

As mentioned before, to prove our platform functionalities, we proposed also a novel solution to use distribution state estimation for active distribution networks in which rooftop PV panels are being increasingly installed in the low voltage grid, while no wide area measurement is available. There are currently several methods used in distribution state estimation of active networks; however, these methods need sparse real-time measurements, which requires a communication infrastructure with large bandwidth and high reliability. Most current networks do not have wide area measurements, while they are rapidly accommodating more and more distributed generations, especially rooftop PV panels in low voltage residential grids. The novelty of our proposed distribution state estimator is to monitor these evolving smart grids during the transitory period when smart meters are not widely deployed in the system for collecting real-time measurements from PVs.

The rest of the paper is organized as follows: Section 2 reviews relevant literature solutions. Section 3 presents the proposed software architecture for general purpose services in smart grids based on SGAM. To better explain what the general and specific elements are, we map our developed use case to SGAM. Section 4 then introduces in detail our use case solution for wide area monitoring of smart grids with high penetration of PV production, without collecting measurements from secondary substations or prosumers. Section 5 presents both the case study and the experimental results of the use case. Finally, Section 6 discusses the concluding remarks.

\section{Related Work}

In this section, we shortly discuss three main contexts based on them we developed our approaches to present in this paper. Firstly, we introduce Smart Grid Architecture Model as a standard and greatly recommended solution to map and analyse newly developed use cases in smart grids. Secondly, we review some existing advanced co-simulation platforms designed or proposed for smart 
grid studies. Finally, we briefly discuss current approach of distribution state estimation for smart grids with Distributed Generation (DG) and highlight its limitation. The contributions of our work will be then explained referring to the presented literature solutions.

\subsection{Smart Grid Architecture Model}

Smart Grid Architecture Model (SGAM) [2] offers a support to design new use cases in smart grids with an architectural approach by which interoperability viewpoints can be well represented. To design new services compliant with SGAM, the following main questions have to be addressed:

1. What are physical components required?

2. How should the information be exchanged?

3. What data should be communicated?

4. What are the needed functions?

5. What are business and regulatory constraints to be applied?

As shown in Figure 1, these considerations respectively form the five SGAM interoperable layers: (i) Component Layer, (ii) Communication Layer, (iii) Information Layer, (iv) Function Layer and (v) Business Layer. Each layer reports the two dimensions of the Smart Grid Plane: zones and domains. Zones represent the hierarchical levels of power system management: Process, Field, Station, Operation, Enterprise and Market. Domains cover the complete electrical energy conversion chain: Bulk Generation, Transmission, Distribution, Distributed Energy Resources (DER) and Customers Premises.

According to [2], the Component Layer consists of all the physical components playing in a smart grid context including hardware devices, physical entities and infrastructures. The Communication Layer includes all the protocols and mechanisms needed to exchange information among actors. The Information Layer defines information objects and flows, type of data exchanged among actors (e.g., measurements, alarms and commands) and underlying canonical data models. The Function Layer consists of smart services in an advanced distribution management system (e.g., advanced outage management, demand response and distributed energy resources control). Finally, the Business Layer defines business objectives, political and regulatory framework to develop a use case.

\subsection{Co-Simulation Platforms}

This section reviews literature solutions on co-simulation platforms for power systems. In [11], Hopkinson et al. present Electric Power and Communication Synchronizing Simulator (EPOCHS) that links PSCAD/EMTDC electromagnetic transient simulator, Positive Sequence Load Flow (PSLF) electromechanical transient simulator and ns-2 communication simulator [12] through a federated simulation based upon custom interface inspired by IEEE 1516 High Level Architecture (HLA) standard [13]. EPOCHS is a breakthrough technology, since it is the first known co-simulator for realizing simulation of power systems and communication networks.

HLA plays a fundamental role in co-simulation techniques and it is commonly used to (i) synchronize different simulation engine, (ii) manage timings between different simulation environment, and (iii) exchange data between simulators. INSPIRE [14], an HLA based co-simulation platform, has been presented as a solution to interconnect a power system simulator called DIgSILENT PowerFactory, a communication network simulator called OPNET and other continuous time-based application modelled in MATLAB, JAVA, GNU R and C++.

A different approach was used to design GECO [15], which interfaces PSLF and ns-2 through a global event-driven mechanism to manage the co-simulation orchestration. Another platform that exploits an event-driven approach is Mosaik [16], a framework for modular simulation of active components in Smart Grids that adopts a discrete event-based simulation approach to execute models. In [17], Mosaik has been used to address integration of DRTS such as RTDS to address a Power-Hardware-in-the-Loop co-simulation platform. 
Another approach refers to ad hoc co-simulation infrastructure, such as SGSim [18,19]. SGSim combines Omnet++, another communication network simulator, and OpenDSS to simulate and investigate new approaches of Smart Grid applications. Moreover, Bian et al. [20] present a real-time co-simulation platform using Opal-RT and OPNET for analysing Smart Grid performance exploiting the possibility to interface OPNET simulation environment with the real world through Internet Protocol (IP). Venkataramanan et al. [21] present an ad hoc co-simulation framework to design a testbed for microgrid cyber-physical analysis by combining (i) RTDS, (ii) a communication network emulator using Common Open Research Emulator (a.k.a. CORE) and (iii) Open Platform Communication based on FreeOPCUA. A more complex ad hoc approach has been proposed in [22] where JADE, a popular multi-agent platform, has been connected to both PSCAD electromagnetic transient simulator and OPNET.

The presented literature solutions do not follow SGAM that supports the design of services highlighting the interoperability among the actors in the smart grid system. Moreover, most of literature solutions are not easy to use and they are more often monolithic rather than distributed software. Indeed, distributed co-simulation platforms allow even to easily include new software components (e.g., new algorithms or services) in a plug-and-play fashion, making the whole platform scalable and flexible. Finally, literature solutions lack the integration of IoT devices and third-party platforms, such as smart metering architectures [10], that can feed the algorithm to be tested with real-world data, even in (near-) real time.

\subsection{Solutions for Wide Area Monitoring}

Wide area monitoring of modern distribution systems is a crucial application in distribution management systems (DMS) in which Distribution system State Estimator (DSE) plays a very important role. The state estimator provides a real-time estimate of the present operating state using the system model and a set of measurements obtained from meters. It provides an estimation of node voltages, branch currents, active and reactive power injections and power flows in all parts of the system.

In traditional distribution systems, where no distributed generation (DG) exists, the voltage and current measured at the head of the feeders are the only available measurements to be used for wide area monitoring. In these networks, conventional distribution state estimation methods could accurately estimate the whole network state. However, vast integration of DGs is shifting the operation of traditional networks from a passive system towards active networks. Although, from many aspects, this transition provides lots of opportunities, but it also creates emerging challenges to the system monitoring and management; conventional methods for state estimation do not work properly or accurately with only measurements from head of feeders.

A solution to this problem is to deploy a wide area measurement system such as Advanced Measurement Infrastructure (AMI), supported by many newly installed smart meters. Despite the various methods proposed $[23,24]$, due to the limited number of measurements downstream from the substations, only a limited number of utility companies have been implemented the state estimation [23]. This means along with growing penetration of DGs, more and more measurement devices should be installed in the network to efficiently deploy state estimation. However, different fields of smart grid technologies do not evolve with the same pace. This has led to current distribution systems with a high rate of DG integration (mainly Photovoltaic in low voltage grids), but no commensurate monitoring infrastructure.

There are a number of methods proposed in the literature for distribution networks with DER $[25,26]$. However, such methods need sparse real-time measurements, which requires a communication infrastructure with large bandwidth and high reliability [27]. The problem is that the current distribution networks are in a transition period towards active distribution networks which is not well supported by developed measurement and communication infrastructures.

In this context, we need a solution for this transitory period to support wide area monitoring of these active networks, independently from wide area measurements. 


\subsection{Contribution}

Referring to the three main topics discussed in the previous sections, we discuss hereafter the scientific novelty of our solution.

The proposed co-simulation infrastructure aims at easing tests and validations of general-purpose services to realize distributed intelligence in a realistic laboratory environment. Differently from literature solutions, it has been designed to be distributed and fully compliant with SGAM so that developers can easily deploy their new components (i.e., new services or simulation modules) in a plug-and-play fashion and assess the interoperability with the other actors (either hardware or software) playing in the smart grid. On the one hand, prior to deploying a newly developed software algorithm or hardware prototype in the real smart grid, it is recommended to map and describe it in SGAM to evaluate its interoperability; on the other hand, any new installation should be supported by some ex-ante lab tests and validations which require simulations; reviewing literature, we found some gap to link the two requirements. Therefore, we developed a laboratory-based co-simulation platform which follows SGAM structure and eases interoperability analysis of new use cases. To allow the communication among its component, our platform exploits IoT communication paradigms and technologies which makes the platform modular, scalable, and flexible [28].

In its core, our co-simulation platform integrates both a DRTS (i.e., OPAL-RT) and an ICT network simulator (i.e., OMNeT++ [9]). DTRS simulates power grids allowing also flexible and scalable software in-the-loop and hardware in-the-loop techniques, whilst the ICT network simulator is needed to study the impact of new Internet-connected technologies (i.e., IoT devices) on the communication networks, evaluating their performance and congestions (e.g., time-delay and packet losses). In addition, our platform provides distributed software components (i) to integrate and manage heterogeneous devices, (ii) to collect data, (iii) to define categories, properties and relations between the actors and (iv) to describe information flows and data models. These features allow interconnecting many virtual or physical devices [29], with other simulation modules and/or third-party software (e.g., smart metering architectures [10]) to allow distributed software in-the-loop simulations. Finally, thanks to the adopted IoT communication paradigms, our co-simulation platform is ready to receive real-world data, even in (near-) real time, that can feed the algorithms to be tested.

To demonstrate how our platform could support developing new control, monitoring, or management schemes for smart grids, we designed a new use case for the above-described problem of wide area monitoring in active distribution networks. Some related works were briefly discussed in Section 2.3, but all methods could handle either passive networks using measurements of head of feeders, or active networks using a wide area measurement infrastructure. We developed a scheme, considering the standard requirements of SGAM interoperability layers, to perform state estimation in distribution systems with high Photovoltaic (PV) production without retrieving wide area measurements (i.e., from secondary substations or prosumers). This algorithm uses a PV simulator which estimates PV production in the network and provides additional pseudo-measurement for state estimation.

\section{SGAM-Based Co-Simulation Architecture}

Future smart grid will be complex and distributed systems that will bring new challenges to the system analysis, control and management. In this context, the interdependency and interoperability of the different distributed actors playing in a smart grid requires wide and accurate test and analysis before field implementation.

In this section, we present our proposed co-simulation architecture that follows the SGAM model depicted in Figure 1. It is a distributed infrastructure that has been designed to be flexible and scalable for testing different general-purpose smart grid's services in a plug-and-play fashion. Our architecture exploits Internet-of-Things (IoT) communication paradigms and technologies that allow a fast bidirectional communication among the actors in the smart grid, either hardware or 
software. Figure 2 depicts our co-simulation architecture for each SGAM layer. The rest of this section describes each layer of the proposed co-simulation architecture in more detail.

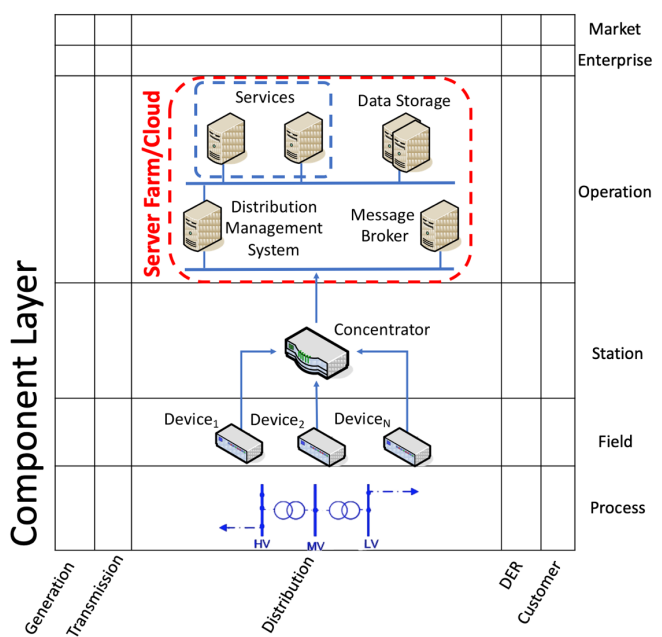

(a) Component Layer

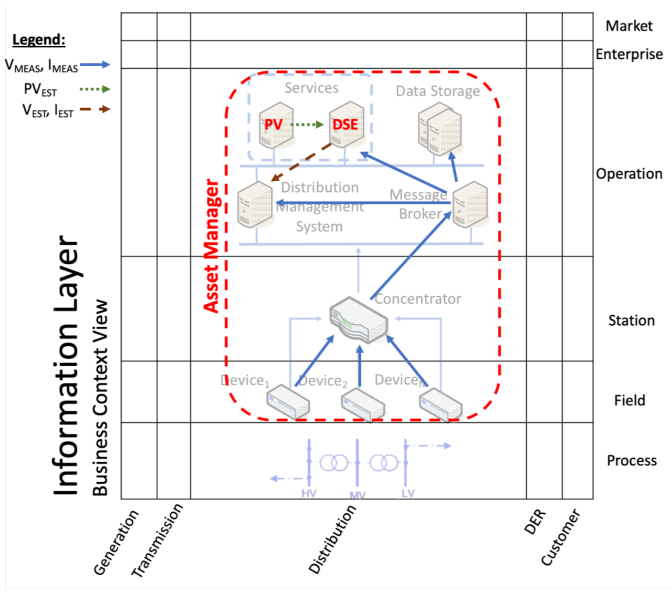

(c) Information Layer: Business context view

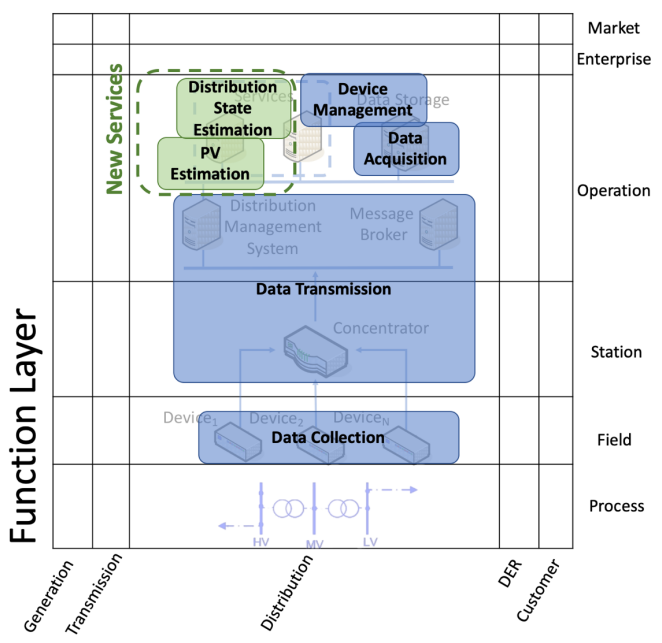

(e) Function Layer

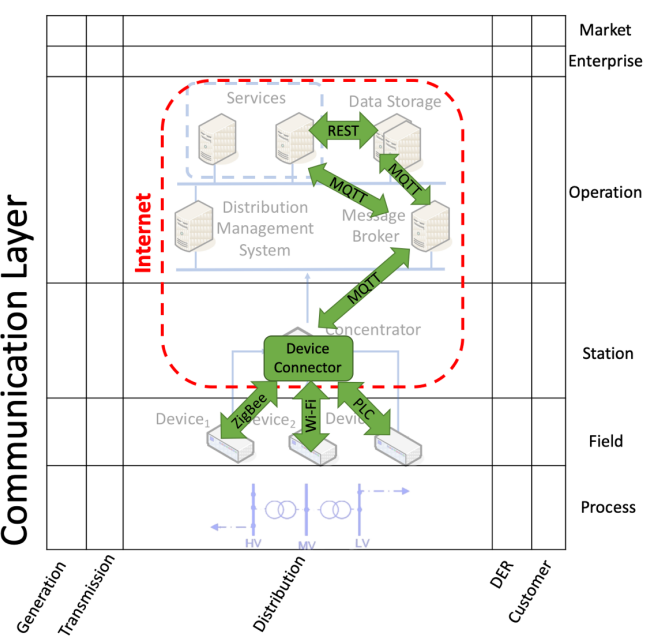

(b) Communication Layer

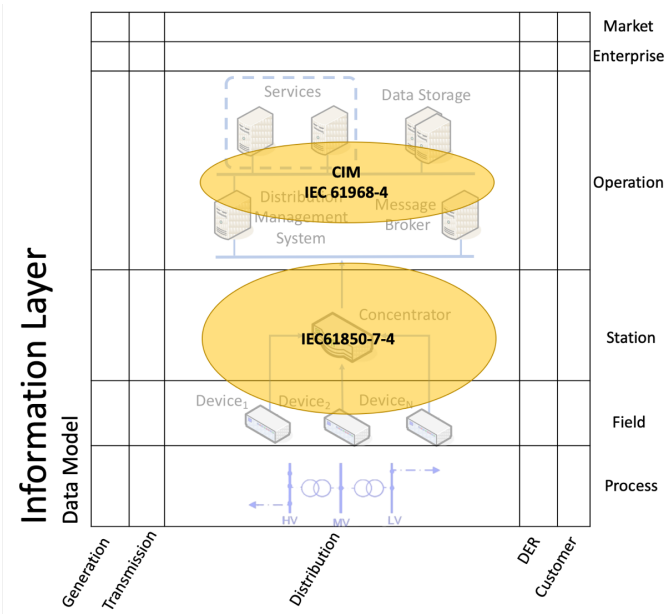

(d) Information Layer: Data model

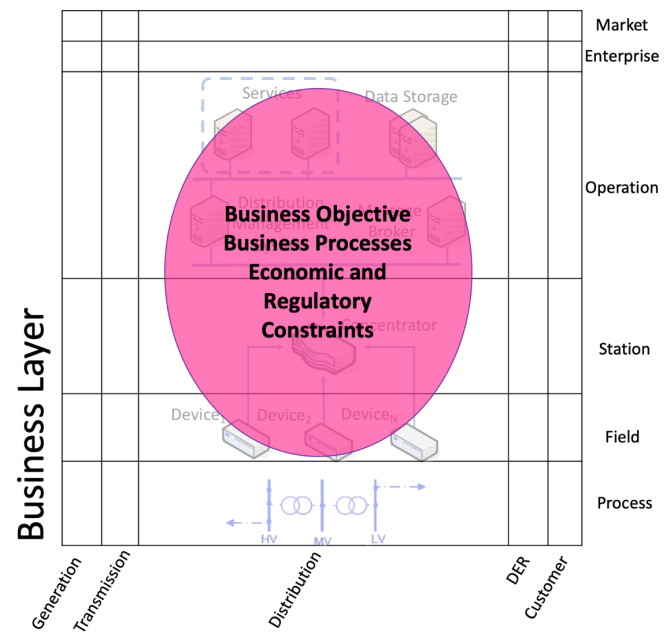

(f) Business Layer

Figure 2. Scheme of the proposed SGAM-based co-simulation architecture. 


\subsection{Component Layer}

The Component Layer (see Figure 2a) includes all the possible hardware technologies that can be installed to perform different services in a distribution network. As shown in the figure, the distribution network is constantly monitored and actuated by a certain number of heterogeneous Devices that exploit a Concentrator to send information and receive commands to/from a clusters of servers where Distribution Management System, Message Broker, Data Storage and Services are deployed. These servers can be physically in a remote server farm or in the cloud. The Distribution Management System is a collection of applications used by the Distribution System Operators (DSO) to monitor, control and optimize the performance of the distribution system and manage it. The Message Broker allows an asynchronous bidirectional communication among the actors in the system through the MQTT (Message Queue Telemetry Transport) protocol [30]. The Data Storage consists of different non-relational databases that are specifically developed for Big Data management ensuring scalability and clusterization. More in depth, our co-simulation architecture implements: (i) InfluxDB [31], an open-source time-series database, to collect measurements from devices and (ii) MongoDB [32], an open-source document-oriented database, to store all the relation between the actors in the system. Finally, Services are all those distributed algorithms needed to maintain and enhance the operational status of the smart grid, such as PV Simulator and Distribution State Estimator (see Section 4).

In our co-simulation infrastructure, the distribution network can be either real or simulated through Digital Real-Time Simulators (DRTS), such as OPAL-RT. DRTS reproduces the behaviour of a real electric distribution system, which is modelled as a testbed for testing and validation of new technologies, management algorithms or control strategies. DRTS allows also to perform software in-the-loop or hardware in-the-loop simulations [33].

For power system electromagnetic transient analysis (EMT), real-time simulation is very reliable with flexibility and scalability due to its computation parallelism feature. All physical components of the electric system can be modelled and simulated by DRTS; this would correspond to the process, field, and station zones in SGAM. However, some of these components like intelligent electronic devices including meters which are normally mapped to the field zone, or data concentrators which are mapped to the station zone could be decoupled from the core of DRTS, in case they are real (physical) devices or prototypes. This so-called hardware in-the-loop experiment enables more complete and real-like testing of the system for interoperability analysis of new devices.

\subsection{Communication Layer}

The Communication Layer includes all the protocols and the software components to allow a fast bidirectional communication over the Internet. As shown in Figure 2b, our co-simulation platform exploits two main protocols for the communication over the Internet: (i) MQTT [30] and (ii) REST (Representational State Transfer) [34], whilst the heterogeneous Devices deployed along the smart grid communicates with different low-level protocols either wireless or wired, such as ZigBee, Wi-Fi or PLC.

MQTT protocol [30] enables an asynchronous bidirectional communication to send data in (near-) real time. It implements the publish/subscribe approach [35]. This paradigm removes the interdependencies between information producer and consumer that increases the scalability of the whole co-simulation platform [28]. This allows for developing distributed services and algorithms that can react to specific events in (near-) real-time ensuring independency from data-sources. On the other hand, REST [34] implements a synchronous communication based on the request/response communication paradigm, which is complementary to publish/subscribe. REST represents a system for transmitting data over the HTTP protocol and it is widely used to develop Web Services.

To integrate the heterogeneous devices in our co-simulation platform and to enable the interoperability among them, we developed different Device Connectors (DeCs). DeC is software component that acts as bridge between our platform and the underlying technologies providing MQTT functionalities, both for publishing and subscribing. In particular, it converts information coming from 
devices into a common data-format adopted by our platform and send it through the MQTT protocol, and vice versa. Each technology needs its own $\mathrm{DeC}$, which is a key component to allow us in using each low-level technology transparently. To develop the different DeCs, we followed a methodology described in [36]. DeCs are installed in the Concentrators, thus, physically closed to the devices to be integrated. In addition, we developed a specific DeC to integrate also DRTS (e.g., OPAL-RT) into our co-simulation platform.

To assess the impact on data transmission according to different Internet media, the Communication Layer includes also OMNeT++ [9], which is a framework to simulate different physical layers and protocols in communication networks (e.g., 5G, 4G and fibre optics). We integrated OMNeT++ in our co-simulation platform following a methodology in $[37,38]$ based on socket connections. In a testbed environment, $\mathrm{OMNeT}++$ can be installed in a server that works as a virtual router that simulates the different network technologies. In this view, all the traffic generated by the actors in the co-simulation platform is routed through our OMNeT++ virtual router to evaluate delays, congestions and packet losses in the communication for each Internet media.

\subsection{Information Layer}

The Information Layer defines categories, properties and relations between the actors, either hardware or software, playing in a smart grid domain (e.g., places, substations and devices). This includes information flows and data models (see Figure $2 c, d$, respectively). This information is georeferenced and is handled by the Asset Manager, which can be installed in a server together with the Data Store where this information is collected.

In case of the proposed wide area measurement-free monitoring system (see Section 4), Figure 2c shows the relations among senders and receivers together with the different information flows. Devices send $V_{M E A S}$ and IMEAS to Distribution Management System, Data Storage and Services through the Concentrator and the Message Broker via MQTT, whilst the Distribution State Estimator (DSE) receives information about PV estimation, $P V_{E S T}$, and provides as output $V_{E S T}$ and $I_{E S T}$ to Distribution Management System via REST.

The actors in the proposed platform exploit the JSON open-standard format to exchange information among them. We choose JSON because it is becoming a common data-format for data exchange in the Web and across IoT devices. It uses human-readable text to transmit data objects consisting of key-value pairs.

Regarding the common information model, our platform is compliant with both IEC 61968 and IEC 61850 standards [39] (see Figure 2d). IEC 61968-4 [40] defines the information model for data exchange between devices and networks in electrical distribution systems, whilst IEC 61850-7-4 [41] defines the communication between devices in transmission, distribution and substation automation systems.

\subsection{Function Layer}

The Function Layer includes all services and algorithms that a specific use case provides. Our co-simulation platform includes the following main functionalities (refers to blue-boxes in Figure 2e): (i) Data Collection performed by Devices to monitor and collect physical behaviours; (ii) Data Transmission to route data and messages from/to the actors in the system through the Internet; (iii) Data Acquisition to store information; (iv) Device Management to remotely manage all the devices deployed in the smart grid. The Device Management features are implemented by the Device Manager, which is another software component in our platform physically installed in a remote server farm or in the cloud.

In addition, this layer provides the functionalities of each individual service for smart grids (refers to green-boxes in Figure 2e). As an example, in case of the proposed wide area measurement-free monitoring system (see Section 4), the platform includes also PV Estimation and Distribution State Estimation to estimate the status of the grid with photovoltaic systems. 
Our platform is flexible in integrating and testing more services and modules (such as new models for renewable energy systems) to realize distributed intelligence. Since this architecture has been designed to be modular, services can either play concurrently or be replaced in a plug-and-play fashion. Thanks to the IoT communication paradigms and technologies adopted to exchange data among the actors in the platform, services can be assessed in testbed environments by exploiting DRTS, or even in real-world scenarios by using real Devices deployed across the smart grid. Replacements or updates can be done without affecting the rest of the platform.

\subsection{Business Layer}

The Business Layer accommodates the use case business processes, services and organizations. This includes the business objectives, regulatory and economic constraint related to the use case. Figure $2 \mathrm{f}$ represents the zones and domains affected by the use case and consequently influenced by underlying business objectives and economic and regulatory constraints. This layer would include the content, structure, and governance of transactions anticipated to create value by taking advantage of business opportunities [42].

The business layer contains non-functional requirements for implementation. Therefore, we do not associate directly some physical components to run/serve such contents. Nevertheless, a business model needs to establish the content and structure. As an example, for the use case, we developed and demonstrated in this work, the business model in general is "monitoring wide area of distribution grid", and, as a detailed view, it can "monitor system normal state violations or operation interruptions and report to regulator". Wide area monitoring is the basis for many other use cases like Fault Detection, Isolation, Restoration (FDIR) [43] or Volt/VAr control [44]. For the security, some of the following regulations, as examples, are required: NERC CIP [45], BDEW White Paper [46] and ISO 27001/02/19 (Information Security Management) [47].

\section{Wide Area Measurement-Free Monitoring}

In this section, we present a DSE solution for distribution systems in which a high level of generation is assumed by rooftop PV panels installed in low voltage systems. We assume either no dedicated smart meter is measuring PV production, or no real-time information is reported by possible connected meters due to insufficient communication infrastructure. Our proposed solution is able to estimate system state with only conventional measurements at the head of feeders.

The proposed DSE considers the presence of multiple PV units. Nevertheless, there are still substations assumed to be supplying purely passive Low Voltage (LV) grids (with no PV installation). We will demonstrate our algorithm not only performs proper state estimation of nodes with no PV connection, but also monitors the network with an acceptable accuracy during nights when no PV power is injected to the grid.

The proposed method for wide area monitoring mainly relies on accurate estimated PV generations using the technique described in Section 4.1. The proposed DSE employs the predicted PV generations as accurate pseudo measurements to predict the current system state. No new meters are needed to be installed widely in the distribution network to measure operational parameters along the feeders, secondary substations, or at customer premises.

Figure 3 presents the overall conceptual architecture of the proposed scheme. Having the weather forecast and city geographic information of an area, load estimator and PV simulator (PVsim) would forecast the generation and consumption data for that area. The distribution management centre receives the forecasted data and the measurements taken at all feeders' main HV/MV substations. 


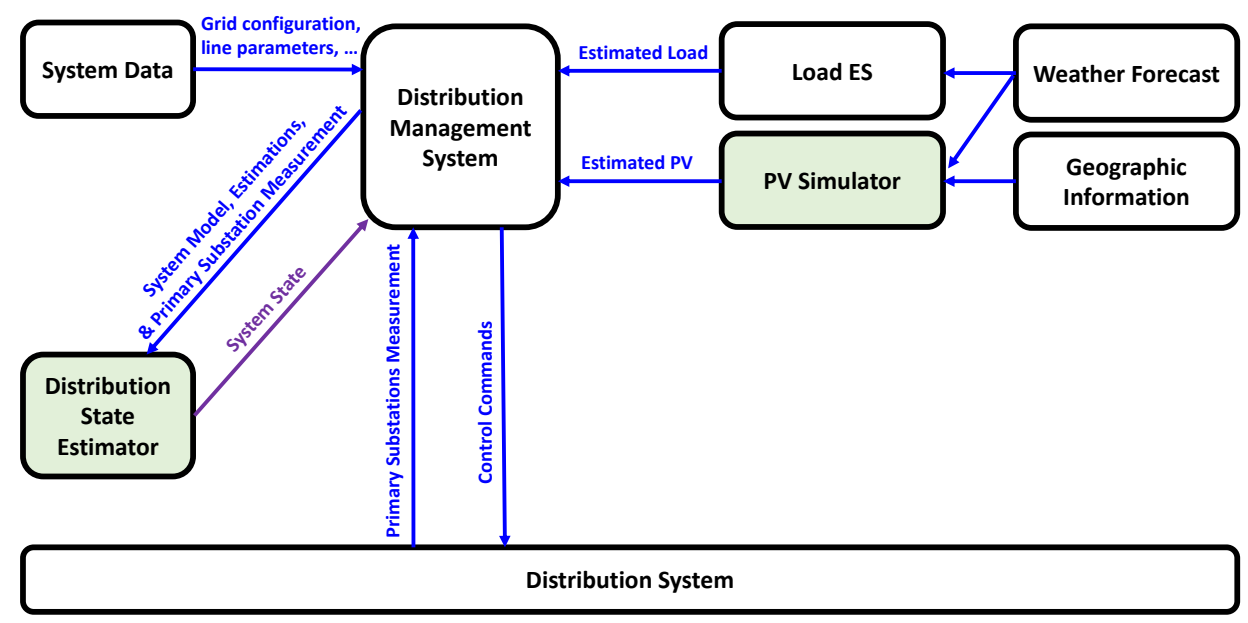

Figure 3. Proposed scheme for wide area monitoring in distribution systems with high PV penetration.

For each time step (e.g., every $15 \mathrm{~min}$ ), having the forecasted consumptions and generations, system electrical data and main substations measurements, the DMS runs the state estimator for each feeder to estimate the system state. With an accurate estimation of the system state for each time step, the distribution system manager can monitor all line currents, node voltages, power losses, etc. in near real time, hence it can send appropriate commands to manage the network.

The scheme components in Figure 3 are the following:

- System data including configuration of the network, parameters of the lines and transformers, and in general information about the grid infrastructure;

- Load ES providing an estimation or forecast of load data;

- $\quad$ PV Simulator giving an accurate estimation of PV production of roof-top PV panels;

- Weather Forecast feeding both Load ES and PV Simulator with climate conditions collected by real weather stations;

- Geographic Information including all the geographic information of the city given as input to PVsim (see Section 4.1);

- Distribution System providing measurements at the beginning of MV feeders in primary substations;

- Distribution State Estimator computing the state of the distribution network;

- Distribution Management System managing the distribution network.

The proposed architecture only requires the system data, estimated demands, estimated PV productions and voltage and current measured at the beginning of MV feeder at the primary substation to predict system state in near real-time. Among these, the system data, load estimates and main substation measurements are already available for traditional distribution networks. The remaining challenges are a method for accurate estimation of PV productions and an appropriate DSE which can realize the proposed architecture in Figure 3. Therefore, the rest of this section introduces PVsim and describes the algorithm for Distribution State Estimator.

\subsection{PV Simulator to Estimate Energy Production}

To estimate energy production of PV systems in urban contexts, we exploited the methodology proposed in our previous work [48] as enabling technology by relying on both GIS (Geographic Information System) and meteorological data.

The main input of the PV simulator (PVsim) is a Digital Surface Model (DSM), our GIS data, which is a high-resolution raster image representing terrain elevation of buildings of interest. Thanks to DSM, 
PVsim detects encumbrances on roofs (e.g., chimneys and dormers) that prevent the deployment of PV panels, and simulates the evolution of shadows on roofs over one year, with 15-minute intervals, in clear-sky conditions. As a preliminary result, this phase identifies the suitable area, which is the real area of a roof that can be used to deploy PV panels.

To estimate the evolution of temperature and irradiance over the time in real-sky conditions, PVsim combines the shadow model with weather data retrieved from weather stations through third-party services (e.g., weather underground [49]). To estimate the incident global radiation, solar radiation is decomposed by considering the attenuation due to air pollution (i.e., Linke turbidity coefficient [50]). Solar radiation decomposition needs as inputs both the direct normal incident radiation and the diffuse horizontal incident radiation [51]. However, if weather stations provide global horizontal radiation only, incident radiation is computed by applying decomposition models in literature [52-57].

Finally, PVsim estimates the PV production for each identified area by using the evolution of temperature and irradiance in real-sky conditions. For this computation, PVsim exploits characteristics and parameters of some commercial PV systems as default values. However, such parameters can be changed according to the characteristics of other commercial PV systems. The final output of the PVsim is georeferenced information on the size of deployable PV systems and the related generation profiles for each building.

\subsection{Distribution State Estimator Formulation}

State estimation refers to the estimation of the most probable values for system state variables using the system mathematical representation and a set of measurements. The calculation of unknown system states is usually done by minimization of a function representing the difference between the estimated and the measured values. The measurement set includes:

1. real measurements (e.g., nodal voltages, branch currents, power flows);

2. pseudo measurements (e.g., the estimated consumption data).

With enough number of measurements which are well distributed over the distribution system, the system will be observable and its state can be estimated. In distribution systems, owing to the large number of pseudo measurements (i.e., loads), observation is rarely a problem.

Measurements are functions of state variables $(x)$. The equations between the state variables $(x)$ and measurements $(z)$ can be presented as follows:

$$
z=h(x)+e
$$

where $h$ is the vector of functions and $e$ denotes the vector of measurement errors. Our Distribution State Estimator is based on Branch current-based State Estimation (BSE), proposed for distribution systems in [58]. In this method, the real and imaginary parts of branch currents are selected as state variables:

$$
x=\left[B_{r}, B_{i}\right]
$$

where $B r$ and $B i$ are the real and imaginary parts of the vector of branch current. The system states can be estimated by minimization of the following least-squares function $j(x)$ :

$$
j(x)=\sum_{i=1}^{m} w_{i}\left(z_{i}-h_{i}(x)\right)^{2}=[z-h(x)]^{T} W[z-h(x)],
$$

where $W$ is the diagonal weighting matrix with $w_{i}$ elements and $m$ is the number of measurements.

The $w_{i}$ elements indicate the influence of each measurement on the resulted estimation. To have more accurate estimations, higher $w_{i}$ values should be considered for more accurate measurements (e.g., nodal voltages) and lower $w_{i}$ values should be considered for inaccurate measurements (e.g., 
estimated loads). Minimizing the least-squares function leads to the best possible values of state variables. By taking the derivation of $j(x)$ and setting it equal to zero, the following iterative solution is obtained:

$$
x^{k+1}=x^{k}+\left[G\left(x^{k}\right)\right]^{-1} H^{T}\left(x^{k}\right) W\left[z-h\left(x^{k}\right)\right],
$$

where the gain matrix $G$ and $H$ are defined as follows:

$$
\begin{gathered}
G(x)=H^{T}(x) W H(x), \\
H(x)=\left[\frac{\partial h(x)}{\partial x}\right] .
\end{gathered}
$$

In the BSE method, by selecting the real and imaginary parts of branch currents as state variables, the $H$ and $G$ matrices become constant which simplifies and speeds up the computations.

During the state estimation, starting from an initial guess, the following equation is solved iteratively until the objective function is minimized, and the state variables are updated in each iteration:

$$
\begin{gathered}
\Delta x_{k}=G^{-1} H^{T} W\left[z-h\left(x_{k}\right)\right], \\
x_{k+1}=x_{k}+\Delta x_{k} .
\end{gathered}
$$

In this paper, as proposed in [58], the real and imaginary parts of branch currents are selected as state variables. Therefore, Equation (7) can be rewritten as follows:

$$
\Delta B_{k}=G^{-1} H^{T} W\left[z-h\left(B_{k}\right)\right],
$$

where $\Delta B_{k}$ denotes the change in branch currents in iteration $k$ and includes a real and an imaginary part:

$$
\Delta B=\left[\Delta B_{r} \Delta \mathrm{B}_{\mathrm{r}}\right]^{T} .
$$

In order to better illustrate the methodology used in our algorithm, the case study is briefly introduced here, while the formulation and procedure is discussed in a generic manner.

The considered distribution system as the case study is shown in Figure 4a. The network is a small portion of an urban medium voltage distribution system in Turin city in the northwest of Italy. A near future scenario of high penetration of roof-top PV production is assumed for low voltage grids supplied by this network. It includes a set of MV feeders feeding customers through MV/LV transformers. The voltages and currents measured at the head of each MV feeder are considered as the only available real measurements, and there is no wide area measurement over this network. The estimated aggregated demands and the aggregated PV generations at MV/LV transformers are considered as pseudo measurements. 


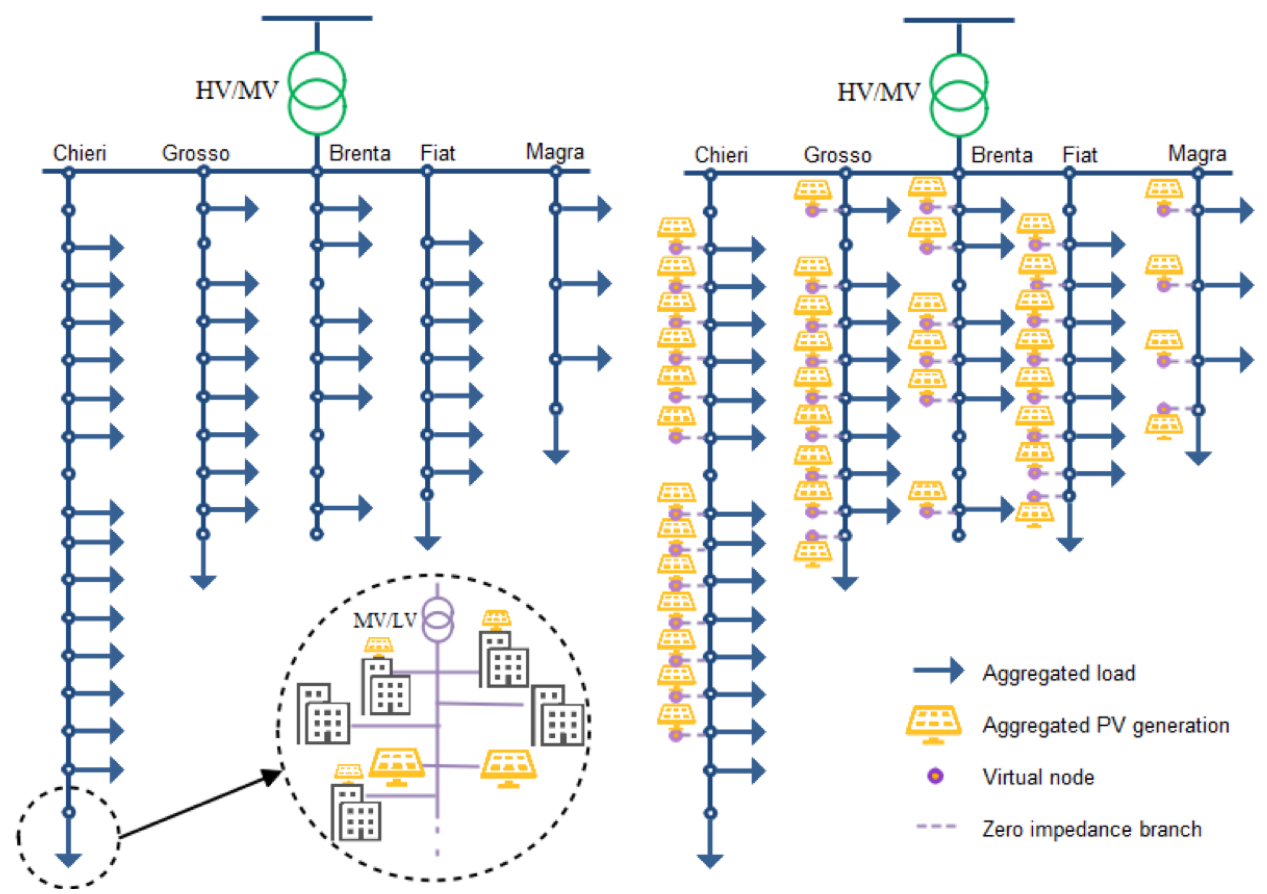

(a) Physical nodes as secondary substations. (b) Virtual nodes for aggregated generations.

Figure 4. MV distribution system with high PV penetration.

For aggregated loads, in each iteration, the estimated load should be converted to an Equivalent Load Current Measurement (ELCM):

$$
I_{k}^{L t}=\left[\frac{P_{L}^{t}+j Q_{L}^{t}}{V_{k-1}^{t}}\right]^{*}
$$

where $P_{L}^{t}$ and $Q_{L}^{t}$ represent the aggregated load for MV/LV transformer at node $t, V_{k-1}^{t}$ is the node voltage at iteration $k-1$, and $I_{k}^{L t}$ is the node equivalent current measurement at iteration $k$.

For aggregated PV generation at each node, similarly, in each iteration, the estimated generation should be converted to an equivalent current measurement:

$$
I_{k}^{G t}=-\left[\frac{P_{G}^{t}+j Q_{G}^{t}}{V_{k-1}^{t}}\right]^{*},
$$

where $P_{G}^{t}$ and $Q_{G}^{t}$ represent the aggregated generation for MV/LV transformer at node $t$, and $I_{k}^{G t}$ is the node equivalent generation current measurement (EGCM) at iteration $k$.

As noted before, the $w_{i}$ elements indicate the influence of each measurement on the resulted estimation. Therefore, to have more accurate estimations, different $w_{i}$ values should be considered for more aggregated loads and aggregated generation based on their estimation accuracies.

The problem is in conventional BSE; it is not possible to consider different weighting values for different equivalent current measurements connected at the same node, while, in our case study, the equivalent model of aggregated PV generators and loads of low voltage systems are connected to the same MV node.

To tackle this challenge, the aggregated generations are considered to be connected to a virtual node, as shown in Figure 4b. The virtual node is then connected to the main MV node using a zero-impedance branch. 
Using the above equations, all the pseudo measurements can be converted to equivalent current measurements and Equation (9) can be rewritten as follows for three phase $(a, b, c)$ :

$$
\left[\begin{array}{c}
\Delta B_{a}^{r} \\
\Delta B_{b}^{r} \\
\Delta B_{c}^{r} \\
\Delta B_{a}^{i} \\
\Delta B_{b}^{i} \\
\Delta B_{c}^{i}
\end{array}\right]^{k}=G^{-1} H^{T} W\left[\begin{array}{c}
\Delta I_{a}^{r} \\
\Delta I_{b}^{r} \\
\Delta I_{c}^{r} \\
\Delta I_{a}^{i} \\
\Delta I_{b}^{i} \\
\Delta I_{c}^{i}
\end{array}\right]^{k},
$$

where $\Delta I$ indicate the difference between the calculated currents and the equivalent current measurements, and subscribes $r$ and $i$ show the variables real and imaginary parts. Figure 5 summarises the method procedure as a flowchart.

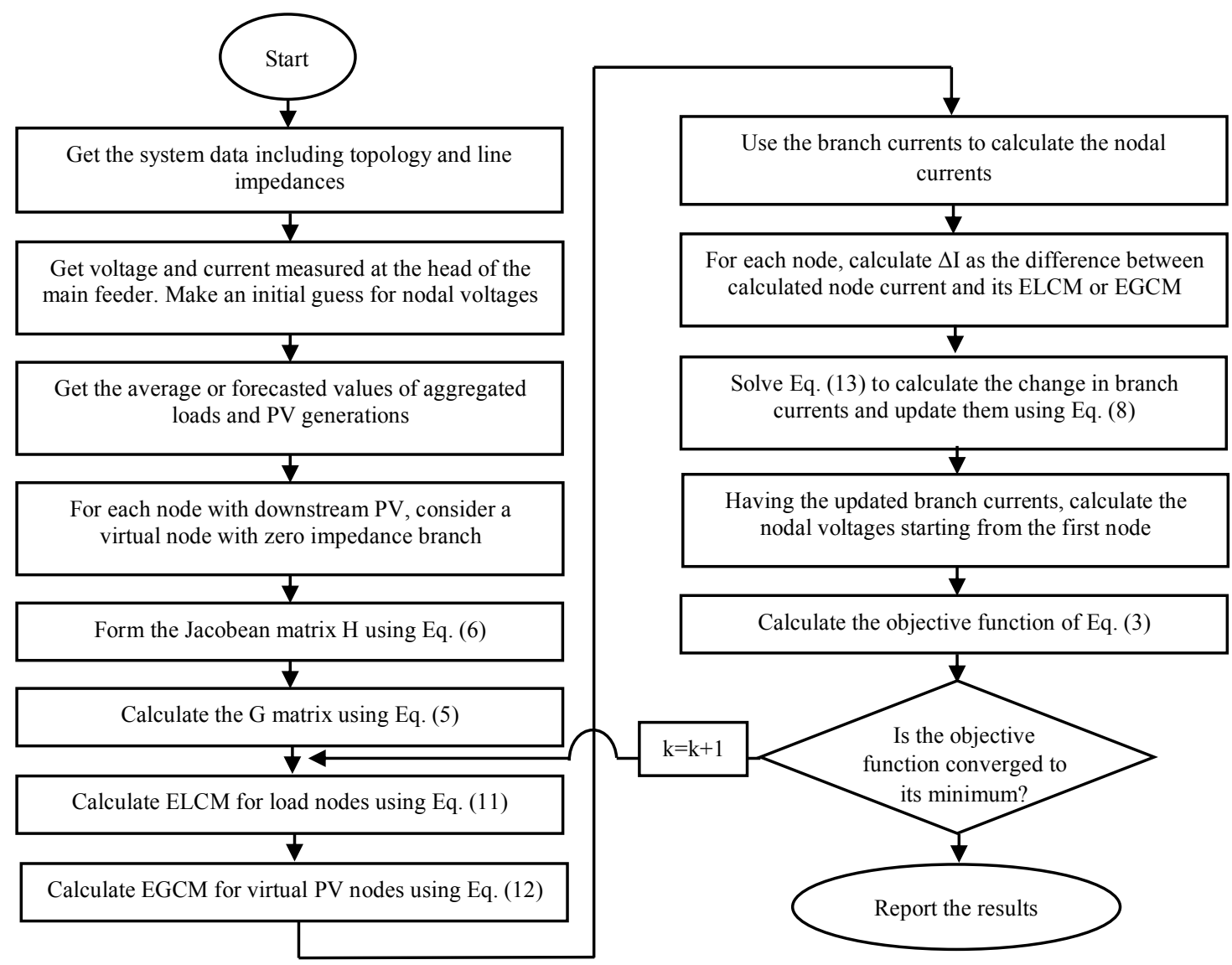

Figure 5. Flowchart of the proposed BSE state estimation.

\section{Case Study and Experimental Results}

To test the performance of the developed algorithm of wide area monitoring discussed in Section 4, we performed some simulations on a portion of an urban distribution system in Turin, city in the northwest of Italy. It should be noted that wide area monitoring requires a wide area measurement collection system like Advanced Metering Infrastructure for a very precise observation of the system operational state. However, currently integration of renewable energy resources, like PV panels in Turin, is growing faster than deploying new measurement devices. Therefore, a solution for the transitory period is needed for wide area monitoring. The results of the proposed scheme may not be as precise as advanced measurement-based schemes, but we demonstrate in this section that it can provide fairly accurate results. 
To perform a real-time simulation, we used an Opal RT digital real time simulator with eMEGAsim configuration of solver which is based on electromagnetic transient analysis (EMT). The modelling environment for eMEGAsim is MATLAB Simulink, and an interfacing software named RT-LAB is used to compile the model to load and execute on hardware simulator. The portion of the real system used in our study is in medium voltage; however, the values of loads and PVs are aggregated power of all low voltage connected prosumers under each secondary substation (MV/LV substation). This network has five MV feeders which are all cables. Hence, we modelled all branches with pi model of transmission lines. It should be noted that the real system is modelled in Opal-RT for real time simulation which plays the role of a real-world system. However, this grid is also modelled in MATLAB for running power flow used in BSE [59]. The former is fed by real and accurate input, while the latter in distribution state estimation gets load and PV input data with errors.

As introduced in Section 4.2, the network in Figure 4 is assumed to be under high PV penetration, and two scenarios of sunny and cloudy days are considered. The co-simulation platform set-up, as shown in Figure 6, includes an OP5600 Opal-RT digital real time simulator to run the grid model including components of process, field and station zones; a laptop PC to run the distribution state estimation algorithm and three different desktop PCs to execute PVsim, to run the MQTT message broker and to accommodate the data storage, respectively.

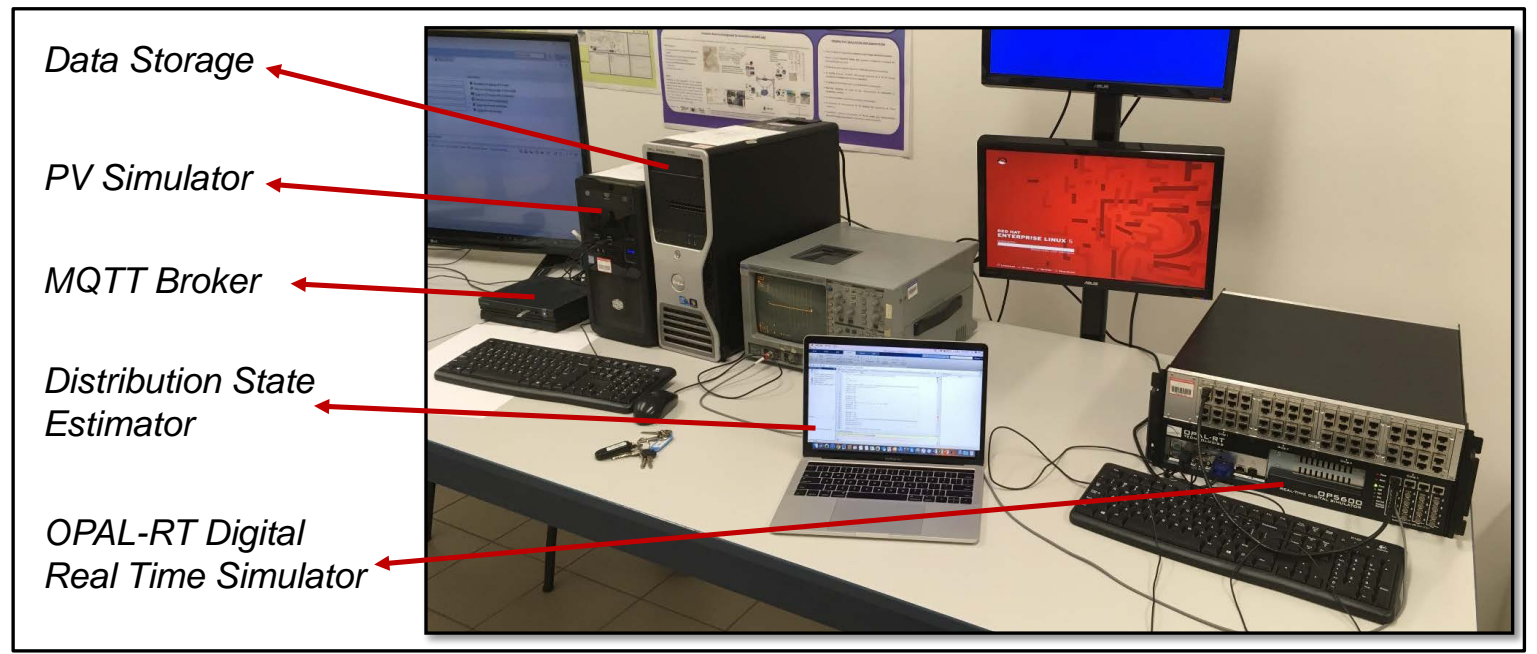

Figure 6. The lab set-up and platform components.

This set-up tries to mimic the real-world system with a real physical network; the real-time simulator can be just replaced with the real network. In the latter case, the real network has to send only measurements from the beginning of feeders at primary substations. From a real-time simulator, this signal is generated by running the virtual model of the grid.

This set-up is actually our test system to observe the results of our new wide area monitoring system which relies on PVsim, rather than wide area measurements. Since no measurements from prosumers are available, the monitoring system is blind to the real amount of production or consumption; we provide just an estimation of consumption with $30 \%$ error.

The reference system to be used for validating the results of test system is actually the real network or the virtual model of the real network with all captured voltage and current values of nodes. In our laboratory, we stored all these values in the real-time simulator and, at the end of each scenario simulation, we made a comparison between these values and what the estimator could estimate. This is illustrated in Figure 7. 


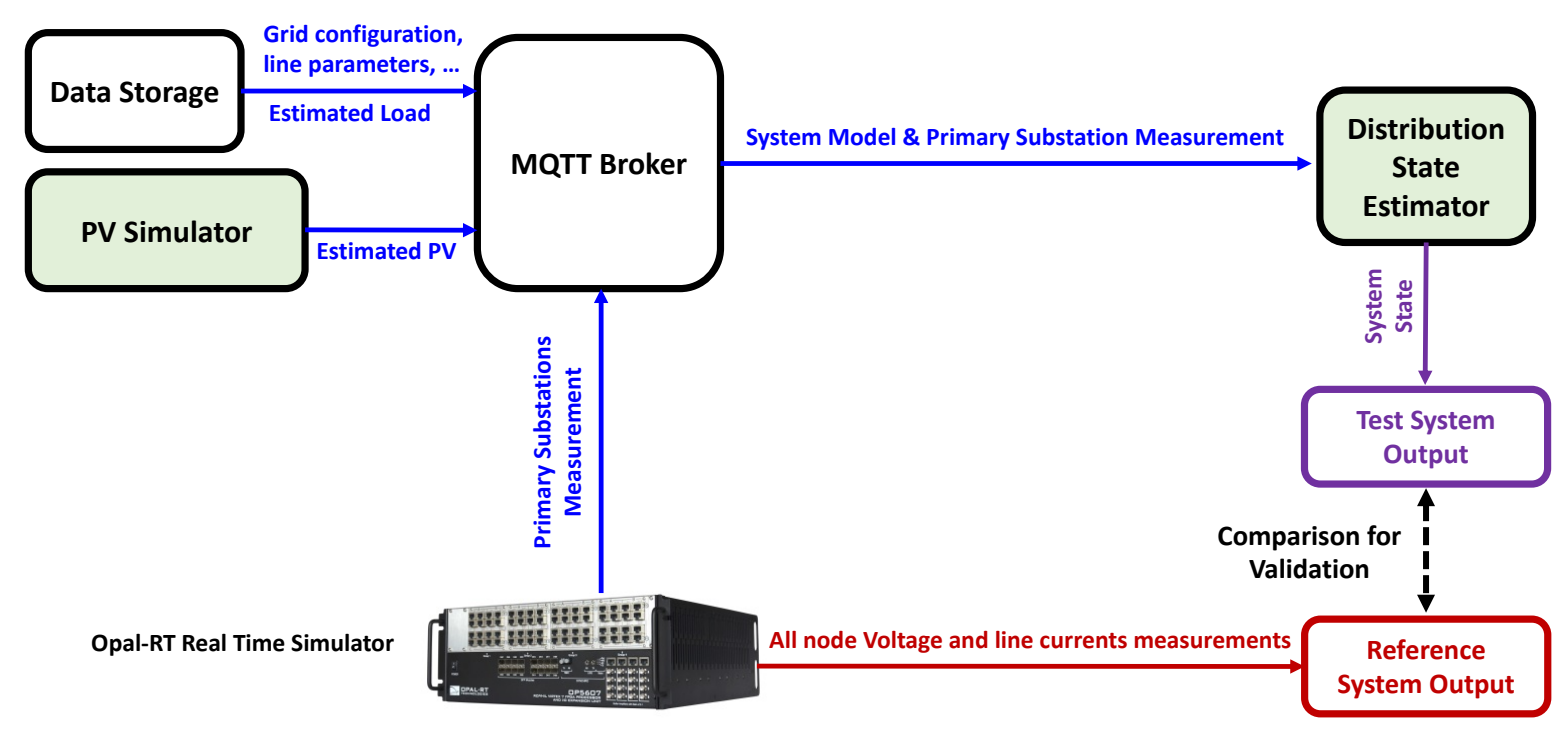

Figure 7. Test and reference system output comparison.

To assess the results of our algorithm for wide area measurements compared to reference data, we exploited the following indexes that are widely used in descriptive statistics:

1. Bias is the mean value of the differences between state estimation and observed values;

2. Mean Absolute Error (MAE) is defined as the average of the absolute difference between the state estimation and the reference data;

3. Root Mean Square Difference (RMSD) is the standard deviation of differences between state estimation and observed values;

4. Correlation Coefficient is a 0 to 1 number representing the strength of the correlation between the state estimation and observed values;

5. Index of Agreement is the standardised measure of the agreement of the state estimation with the observed values. It ranges from -1 to 1 , where 0 means no agreement, -1 a perfect negative agreement and 1 a perfect positive agreement, respectively.

We report Bias, MAE and RMSD in percentage.

Figure 8a shows the maps of the selected substations reporting the involved buildings with their heights, expressed in floors (i.e., the reference case). As shown in the map, most of the buildings are lower than eight floors and just a few of them are higher than 17 floors. In this urban area, we simulated the PV energy production by exploiting the PVsim presented in Section 4.1. We supposed to deploy PV modules with the following characteristics: (i) nominal power of about $283 \mathrm{~W}$, (ii) efficiency in standard condition of $22.5 \%$, (iii) temperature coefficient equal to $0.38[\% / C]$. The total capacity of the whole area is $48.45 \mathrm{MW}$, with a mean value of $1.1 \mathrm{MW}$ for each substation. Figure $8 \mathrm{~b}$ shows the energy produced for each of the 43 substations. As shown in the figure, the maximum energy, about 2.68 MW, is produced by PV systems at substation 10. Figure $9 \mathrm{a}, \mathrm{b}$ present our simulation results for two days with different meteorological conditions: sunny and cloudy, respectively. These figures would refer to our test system. 


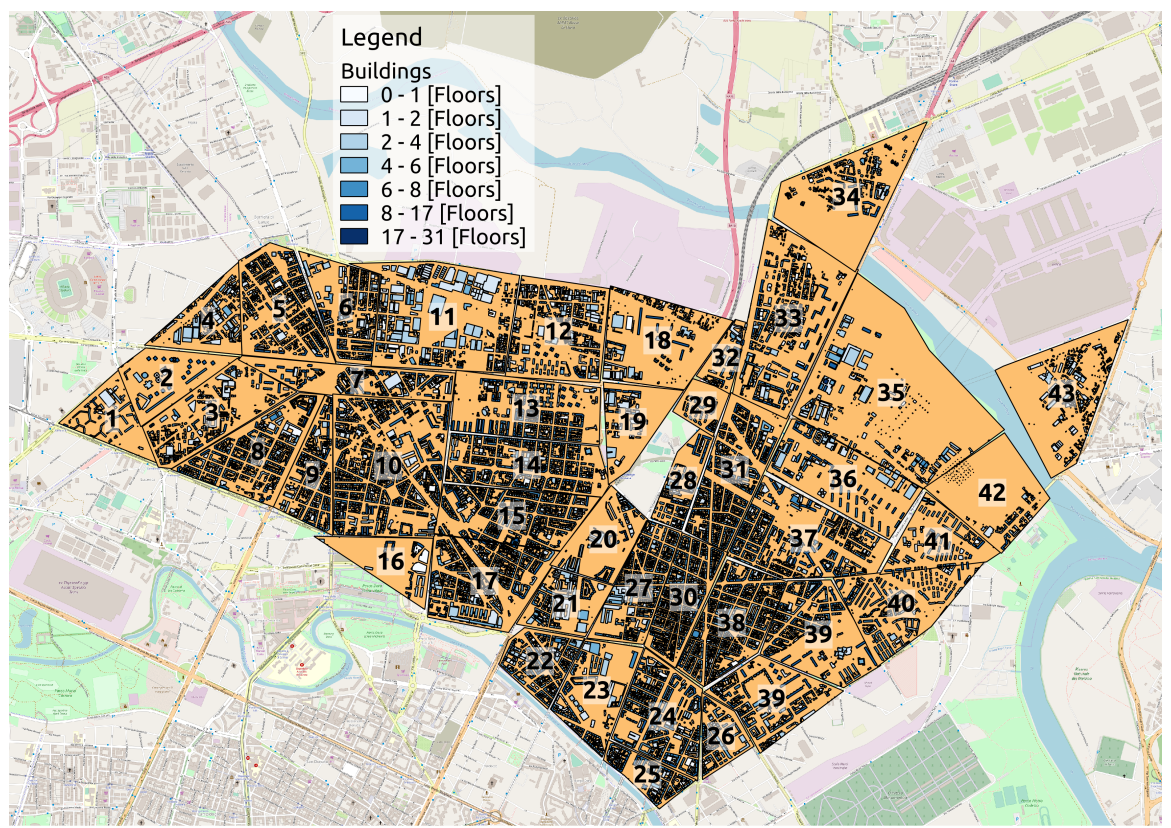

(a) Number of floors per building.

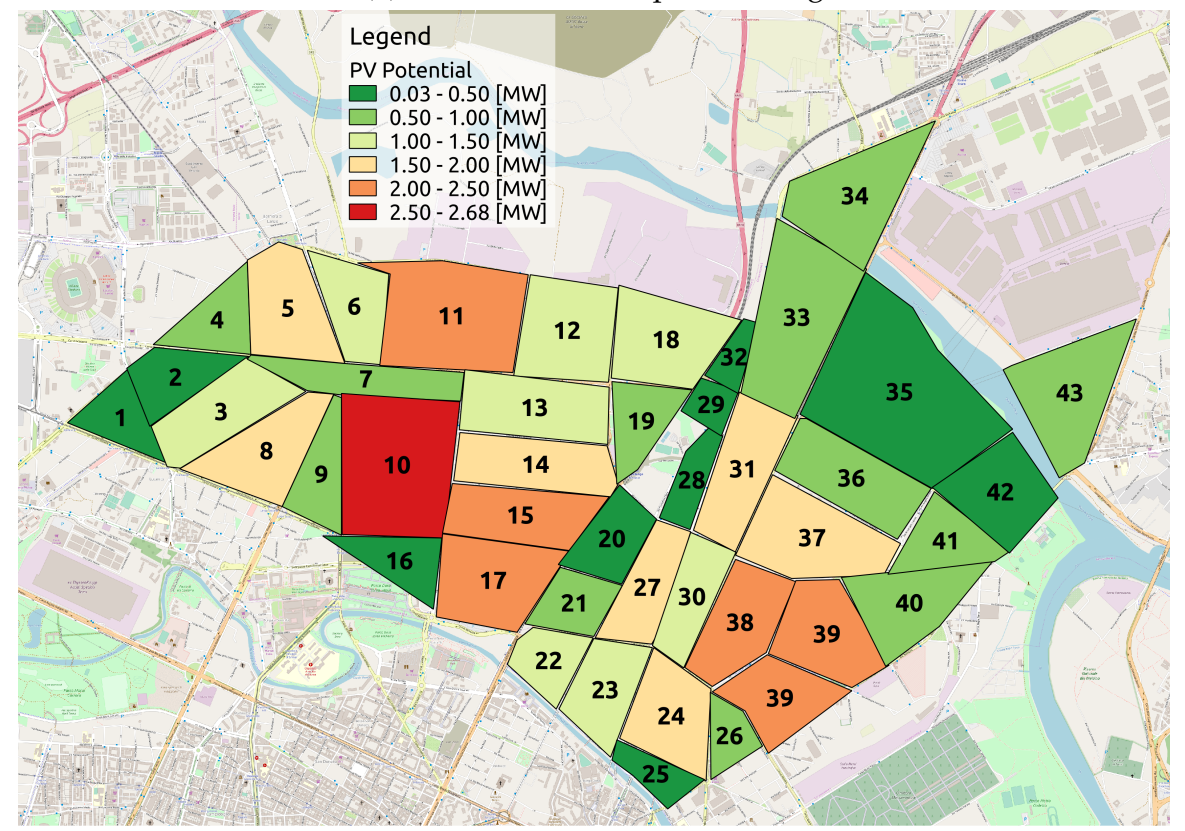

(b) PV potential of the Area.

Figure 8. Case study area. 


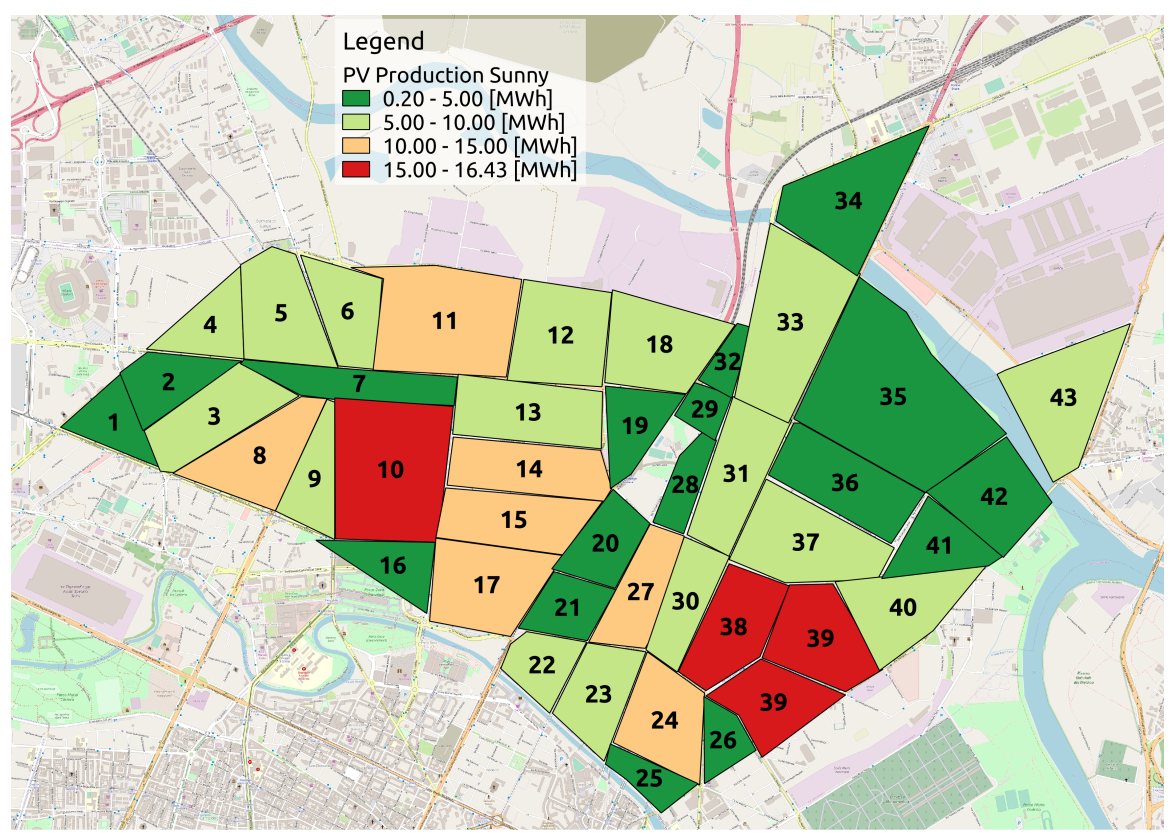

(a) PV Energy Production in a sunny day.

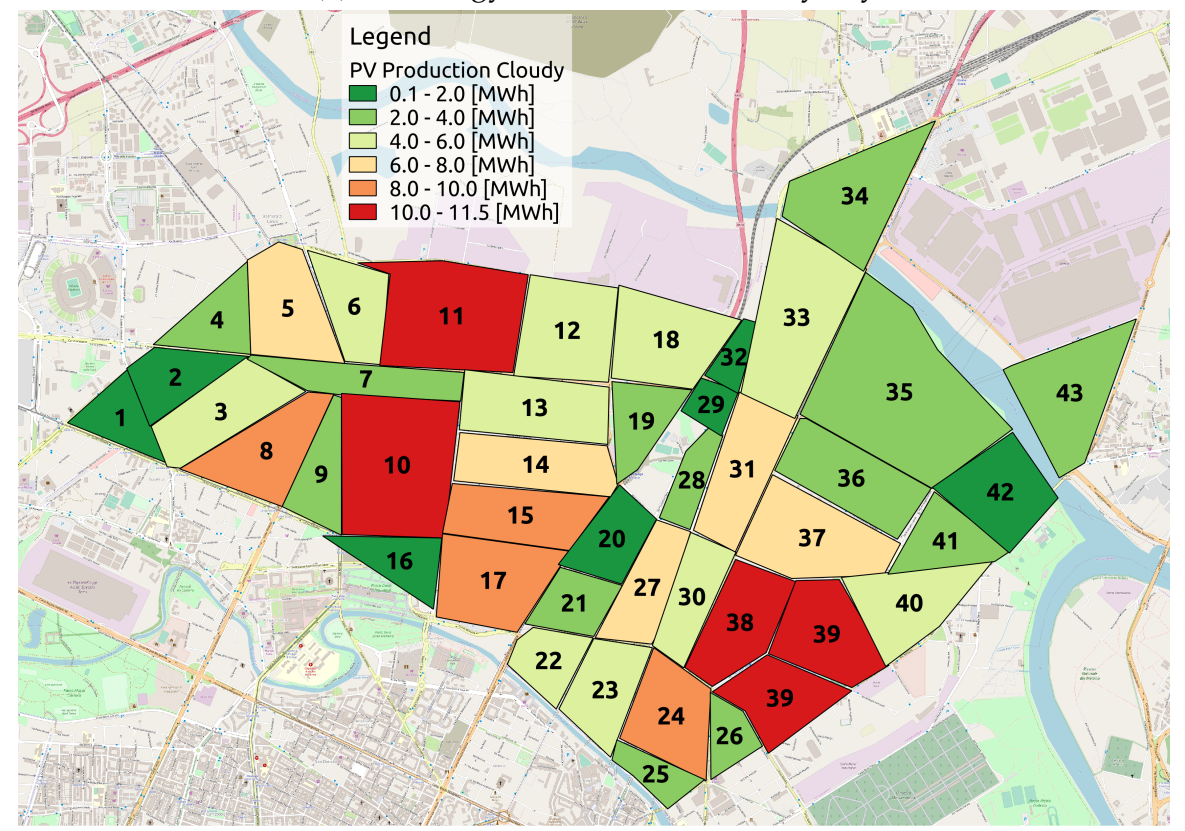

(b) PV Energy Production in a cloudy day.

Figure 9. PV energy production.

Figure 10 shows the map of the daily energy consumption of each substation. This is made based on the reference scenario of consumption with no errors (i.e., the reference system). It is possible to notice that the seven substations have the highest consumption that ranges between 12.4 MWh and 13.3 MWh. Just one substation has a level of consumption lower than 1.8 MWh and the majority of the substations have a consumption ranging between 4.1 MWh and 8.4 MWh.

In our simulations, we supposed that load data has an estimation error of about $30 \%$. Two cases are considered to evaluate the proposed methods performance. In the first case, the test system is considered to be heavily loaded and weather is considered to be sunny. Figure 11 presents the estimated and actual voltage magnitudes of the last node of the test system longest feeder (refers to Chieri feeder in Figure 4). This is because the last node highlights the effect of errors and it is more interesting to be used for validation of the algorithm. As shown in this figure, the estimated voltage 
profile perfectly matches the actual node voltage. The main contribution of the PV units is the middle of the day. In this period while the electricity consumptions are higher, the PV units help the network to keep the voltage levels in the standard range (0.95-1.05 p.u.).

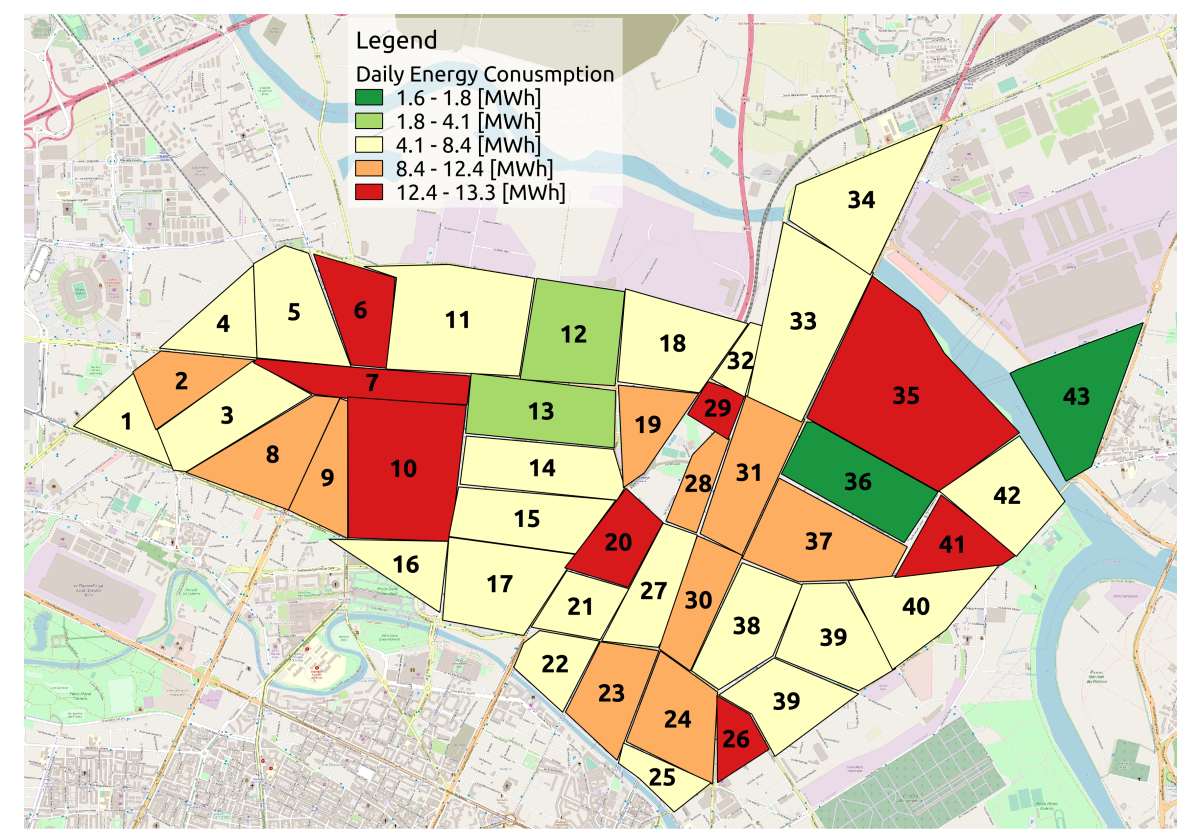

Figure 10. The actual energy consumption of buildings.

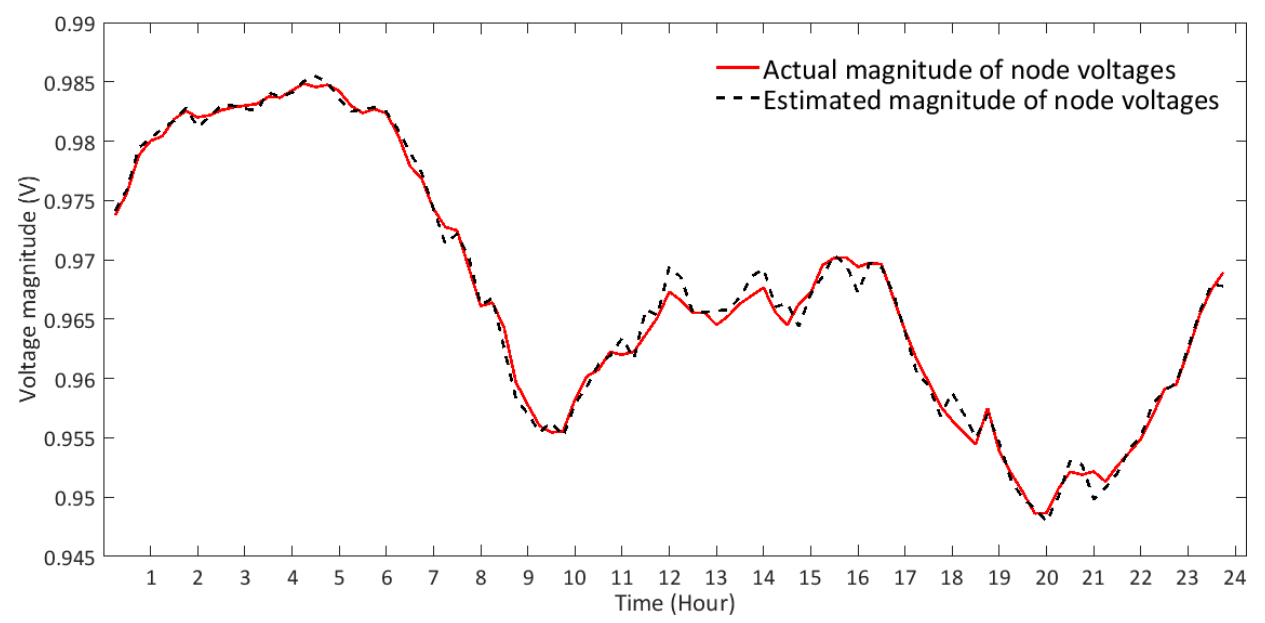

Figure 11. The estimated and actual values of the last node voltage (sunny day).

The same load profiles are considered for the second case for which the weather is considered to be cloudy. The voltage profile of the feeder last node under this case is presented in Figure 12. As can be seen, also for this case, the difference between the estimated and actual voltages is acceptable and the accuracy is very high. This accurate results are confirmed by the performance indexes in Table 1 . Indeed, both Correlation Coefficient and Index of Agreement are 0.99 for both sunny and cloudy days, which indicates a high match between the actual and estimated voltages. It is interesting to note that the lower PV productions in Figure 12 compared to Figure 11, due to cloudy weather, have resulted in under voltage situation for some hours of the day. 


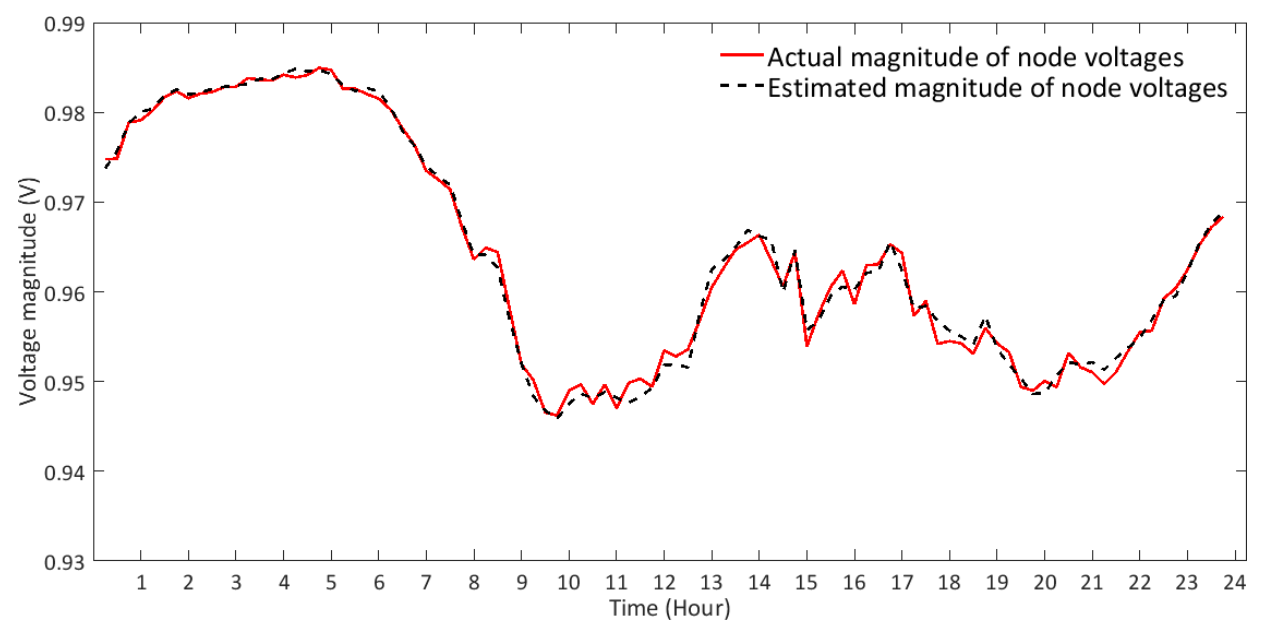

Figure 12. The estimated and actual values of the last node voltage (cloudy day).

Table 1. Performance indexes for estimation.

\begin{tabular}{ccccccc}
\hline & & Bias [\%] & MAE [\%] & RMSD [\%] & $\begin{array}{c}\text { Correlation } \\
\text { Coefficient }\end{array}$ & $\begin{array}{c}\text { Index of } \\
\text { Agreement }\end{array}$ \\
\hline \multirow{2}{*}{ Last node } & sunny day & 0.016 & 0.08 & 0.098 & 0.99 & 0.99 \\
& cloudy day & -0.006 & 0.065 & 0.08 & 0.99 & 0.99 \\
\hline \multirow{2}{*}{ All nodes } & sunny day & -0.008 & 0.036 & 0.055 & 0.99 & 0.99 \\
& cloudy day & 0.005 & 0.03 & 0.049 & 0.99 & 0.99 \\
\hline
\end{tabular}

Node voltages are the parameters usually used to evaluate the accuracy of different state estimation methods. Therefore, the voltage of all nodes of the feeder are presented in Figures 13 and 14 for both the sunny and cloudy day cases at 12:00 p.m. As shown, for both cases, there is a good match between the estimated and actual voltages. Regarding the performance of our distribution state estimation, although the scheme is designed for smart grids under high PV penetration, but even for the nodes with no PV connection, estimations are pretty accurate. These good performances are confirmed by the indexes for all 18 nodes in Table 1, which indicate the overall performance for the whole feeder. Even in this case, both Correlation Coefficient and Index of Agreement are 0.99 for both sunny and cloudy days. Bias ranges between $-0.008 \%$ and $0.005 \%$. MAE and RMSD are about $0.03 \%$ and $0.05 \%$, respectively. Thus, among the nodes in the feeder under analysis, the error rate is about $0.1 \%$, which is very low.

The feeder line currents at 12:00 p.m. for both sunny and cloudy days are presented in Figures 15 and 16 for its 18 lines. For both cases, the system is considered to be heavily loaded. As shown in the figures, there is little difference between the estimated and actual line currents.

In case of sunny days, due to the increase in local power generation, the feeder line currents are decreased. However, in the case of cloudy days, the feeder has high line current values. As can be seen, the PV penetration level has considerably changed the line currents. The same effect can be seen in Figures 13 and 14, where the decrease in local power generation in a cloudy day has resulted in lower voltage levels, compared to a sunny day. 


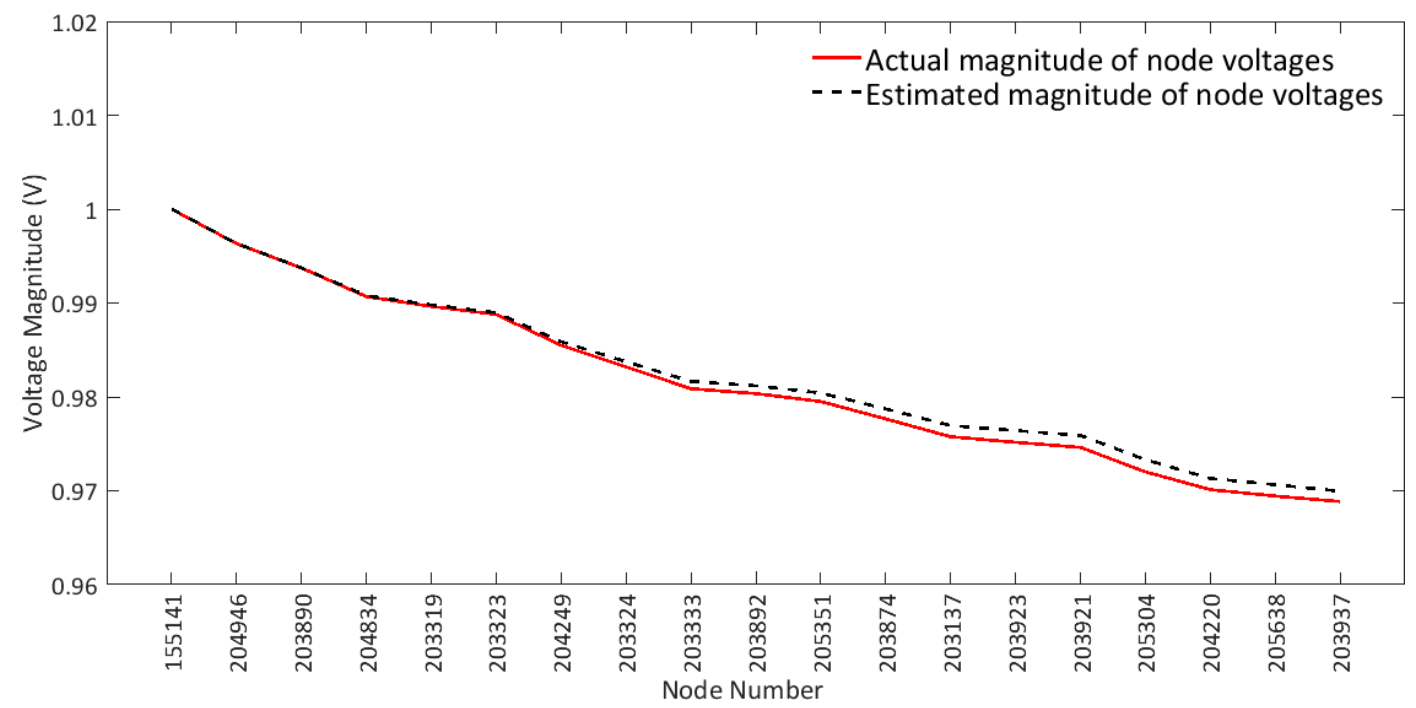

Figure 13. The estimated and actual values of the node voltages at 12:00 p.m. (sunny day).

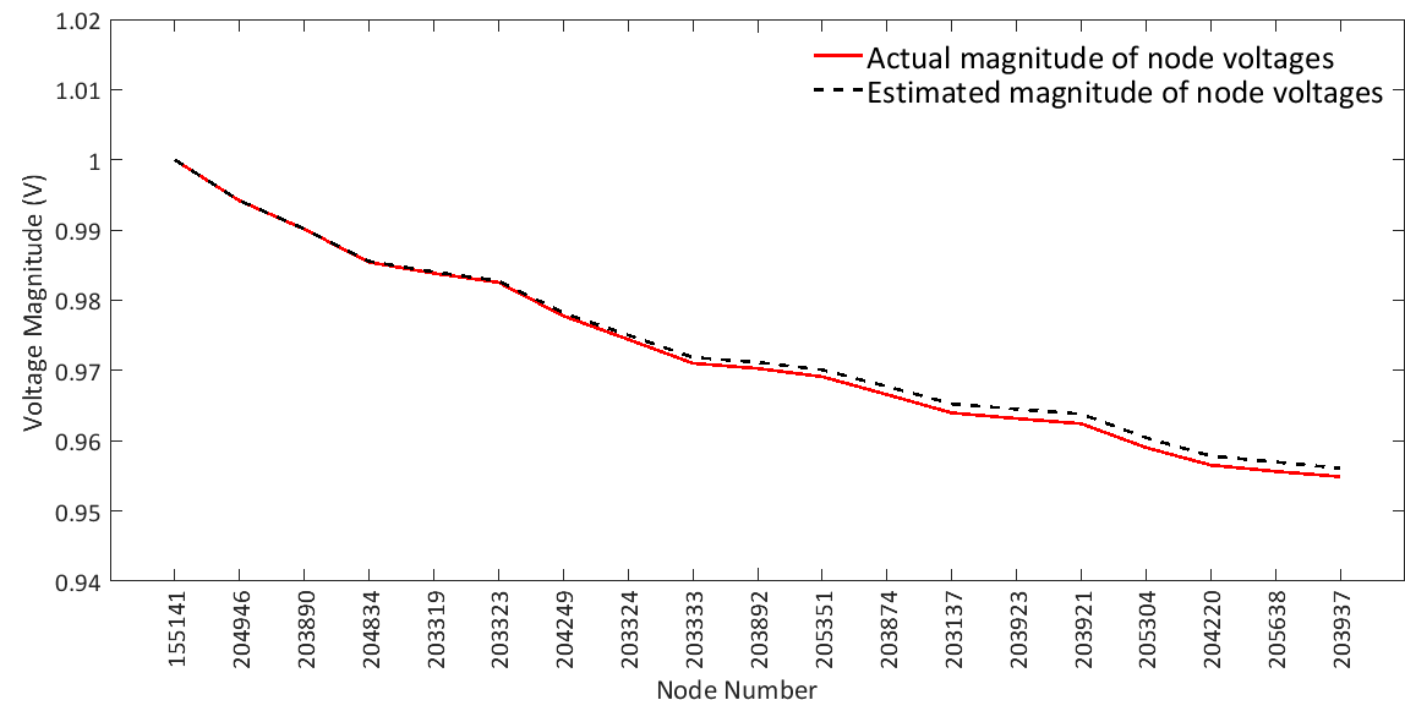

Figure 14. The estimated and actual values of the node voltages at 12:00 p.m. (cloudy day).

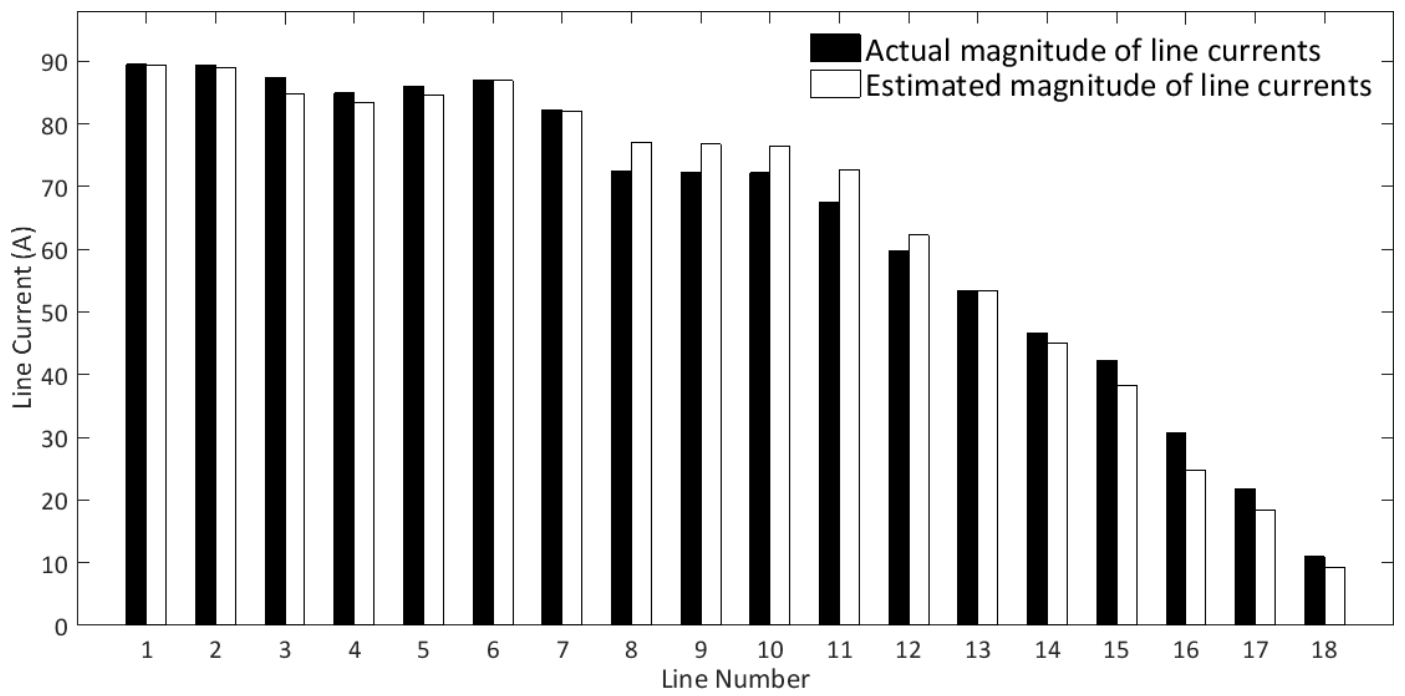

Figure 15. The estimated and actual values of the line currents at 12:00 p.m. (sunny day). 


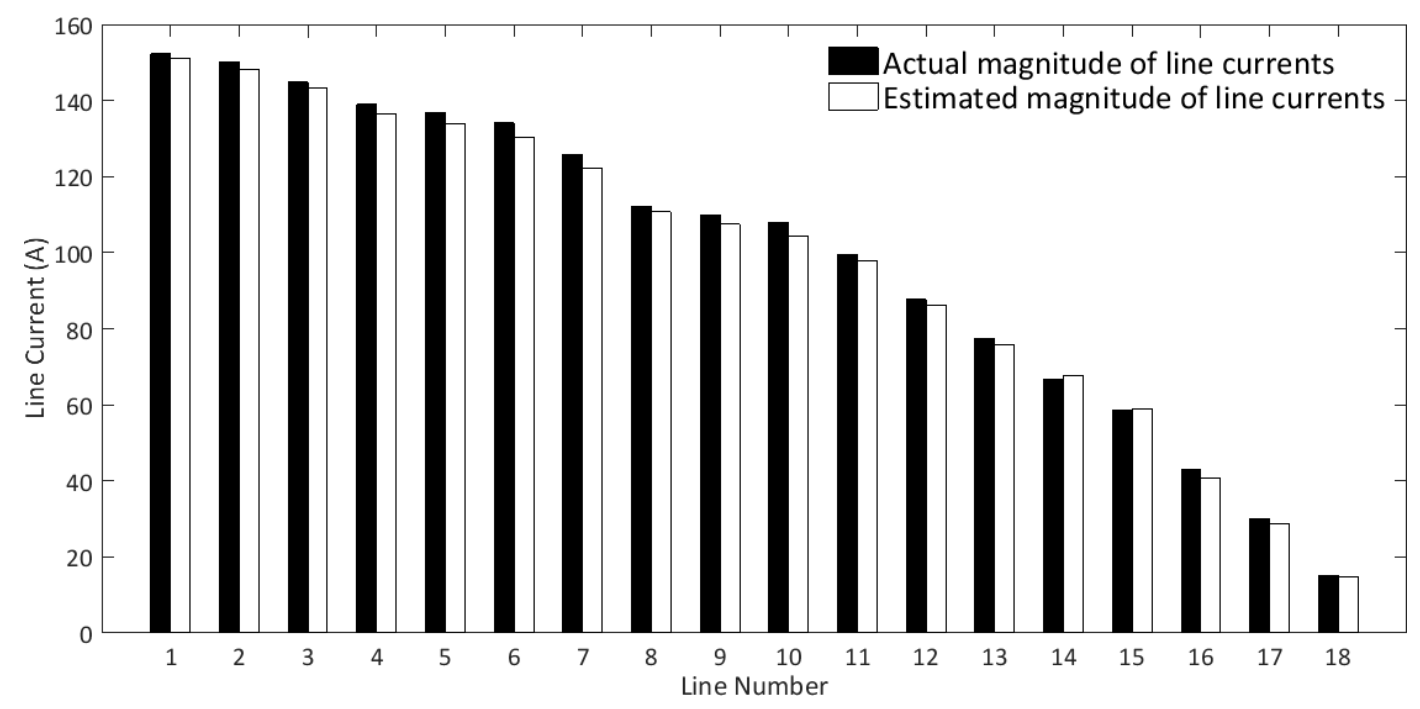

Figure 16. The estimated and actual values of the line currents at 12:00 p.m. (cloudy day).

Such changes caused by variations of local power generation highlight the need for a monitoring scheme for electricity distribution systems with large-scale DG integration but no commensurate monitoring infrastructure. To highlight the potential of the proposed method for better grid monitoring, Figure 17 presents the feeder actual load demands, estimated load demands and the available erroneous load data employed for the estimations. As it can be seen from the plot, the proposed architecture helps making an accurate estimation of the actual system loading. Besides the load demands, estimating the line flows is important for grid control and management. Figure 18 shows the estimated values of line power flows which match the actual line flows with acceptable accuracy.

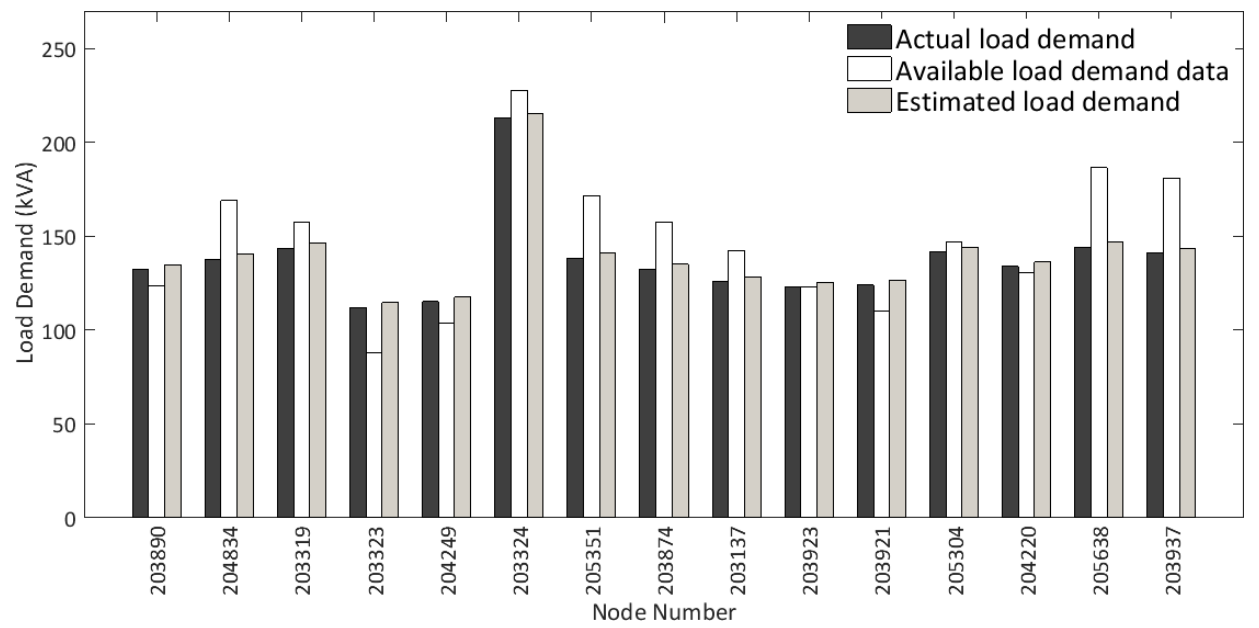

Figure 17. The estimated, actual and available load demands at 12:00 p.m. (sunny day). 


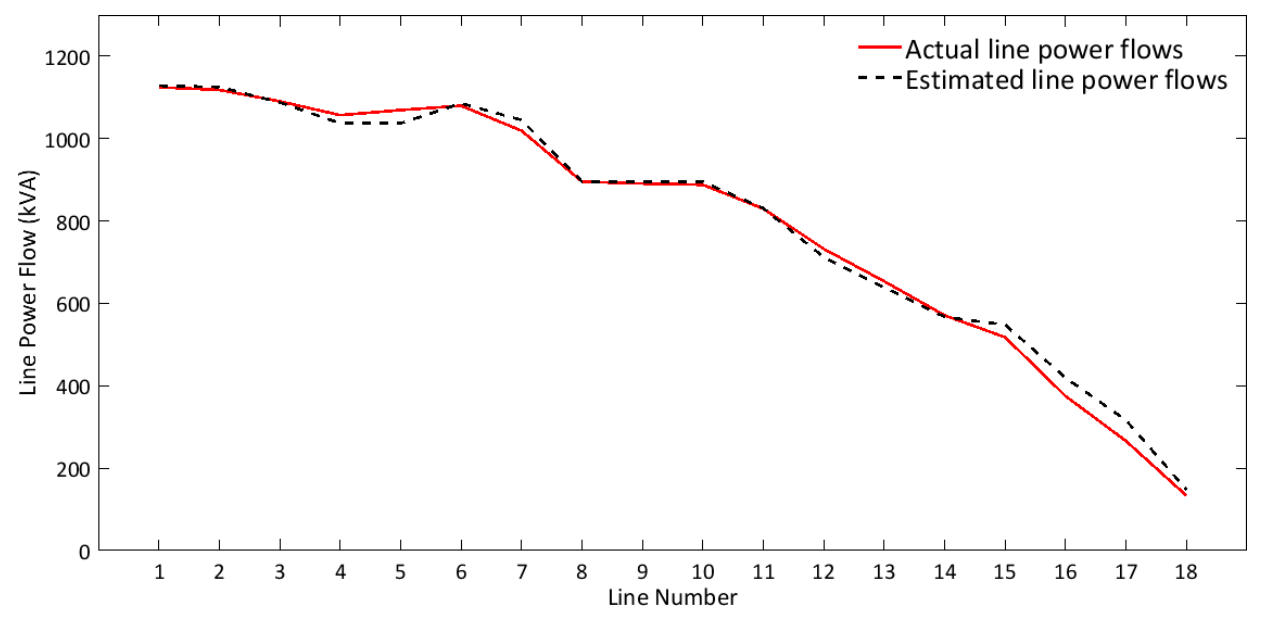

Figure 18. The estimated and actual line power flows at 12:00 p.m. (sunny day).

\section{Conclusions}

To develop new use cases for smart grids, it is wise and also recommended to reuse existing assets as much as possible while shifting towards new control and management paradigms. This would require mapping use cases to SGAM. However, test and validation of newly developed use cases are usually performed in laboratories where interoperability analysis might be overlooked. We demonstrated how an IoT-based infrastructure could support establishing a co-simulation platform following SGAM requirements. Integrating a digital real-time simulator could enable use case developers to test physical devices through hardware in-the-loop set-ups as well as evaluating the performance of new algorithms via software in-the-loop experiments.

As an example of using this platform for developing a use case, a Distributed State Estimator is proposed for monitoring of traditional distribution systems with high PV penetration without a massive deployment of new IoT devices. The experimental results, performed in real MV Distribution system with high PV penetration, demonstrated that the proposed DSE has good performance either in sunny or cloudy days, with an error rate lower than $1 \%$ and a high correlation, about 0.99 , between our results and reference data. Though the results of the proposed monitoring scheme architecture are not as precise as a real advanced measurement infrastructure, it can provide fairly accurate results. It does not require any new installations and can be considered as a cheap and practical solution for the current transition period of distribution systems with increasing penetration of PV units.

Author Contributions: Conceptualization, A.E. and L.B. (Luca Barbierato), A.B. and L.B. (Lorenzo Bottaccioli), E.M. and E.P.; Data curation, A.E. and L.B. (Luca Barbierato), A.B. and L.B. (Lorenzo Bottaccioli) and E.P.; Formal analysis, A.E., A.B. and L.B. (Lorenzo Bottaccioli); Investigation, A.E., A.B. and L.B. (Lorenzo Bottaccioli) and E.P.; Methodology, A.E. and L.B. (Luca Barbierato), A.B. and E.P.; Software, L.B. (Luca Barbierato) and L.B. (Lorenzo Bottaccioli) and E.P.; Supervision, A.E., E.M. and E.P.; Writing-original draft, A.E. and L.B. (Luca Barbierato), A.B., L.B. (Lorenzo Bottaccioli) and E.P.

Funding: This research received no external funding.

Conflicts of Interest: The authors declare no conflict of interest.

\section{References}

1. Farhangi, H. The path of the smart grid. IEEE Power Energy Mag. 2010, 8, 18-28. [CrossRef]

2. Bruinenberg, J.; Colton, L.; Darmois, E.; Dorn, J.; Doyle, J.; Elloumi, O.; Englert, H.; Forbes, R.; Heiles, J.; Hermans, P.; et al. CEN-CENELEC-ETSI Smart Grid Co-Ordination Group Smart Grid Reference Architecture. Available online: https:/ / ec.europa.eu/energy/sites/ener/files/documents/xpert_group1_ reference_architecture.pdf (accessed on 20 February 2019).

3. Podmore, R.; Robinson, M.R. The role of simulators for smart grid development. IEEE Trans. Smart Grid 2010, 1, 205-212. [CrossRef] 
4. Dufour, C.; Bélanger, J. On the use of real-time simulation technology in smart grid research and development. IEEE Trans. Ind. Appl. 2014, 50, 3963-3970. [CrossRef]

5. Guo, F.; Herrera, L.; Murawski, R.; Inoa, E.; Wang, C.L.; Beauchamp, P.; Ekici, E.; Wang, J. Comprehensive real-time simulation of the smart grid. IEEE Trans. Ind. Appl. 2013, 49, 899-908. [CrossRef]

6. Cintuglu, M.H.; Mohammed, O.A.; Akkaya, K.; Uluagac, A.S. A Survey on Smart Grid Cyber-Physical System Testbeds. IEEE Commun. Surv. Tutor. 2017, 19, 446-464. [CrossRef]

7. Palensky, P.; Meer, A.A.V.D.; Lopez, C.D.; Joseph, A.; Pan, K. Cosimulation of Intelligent Power Systems: Fundamentals, Software Architecture, Numerics, and Coupling. IEEE Ind. Electron. Mag. 2017, 11, 34-50. [CrossRef]

8. Palensky, P.; van der Meer, A.; Lopez, C.; Joseph, A.; Pan, K. Applied Cosimulation of Intelligent Power Systems: Implementing Hybrid Simulators for Complex Power Systems. IEEE Ind. Electron. Mag. 2017, 11, 6-21. [CrossRef]

9. OMNeT++, Discrete Event Simulator. Available online: https://www.omnetpp.org/ (accessed on 4 April 2019).

10. Pau, M.; Patti, E.; Barbierato, L.; Estebsari, A.; Pons, E.; Ponci, F.; Monti, A. A cloud-based smart metering infrastructure for distribution grid services and automation. Sustain. Energy Grids Netw. 2018, 15, 14-25. [CrossRef]

11. Hopkinson, K.; Wang, X.; Giovanini, R.; Thorp, J.; Birman, K.; Coury, D. EPOCHS: A platform for agent-based electric power and communication simulation built from commercial off-the-shelf components. IEEE Trans. Power Syst. 2006, 21, 548-558. [CrossRef]

12. The Network Simulator-ns-2. Available online: https://www.isi.edu/nsnam/ns/ (accessed on 4 April 2019).

13. IEEE 1516-2010-IEEE Standard for Modeling and Simulation (M\&S) High Level Architecture (HLA)-Framework and Rules. Available online: https://standards.ieee.org/standard/1516-2010.html (accessed on 4 April 2019).

14. Georg, H.; Müller, S.C.; Rehtanz, C.; Wietfeld, C. Analyzing Cyber-Physical Energy Systems: The INSPIRE Cosimulation of Power and ICT Systems Using HLA. IEEE Trans. Ind. Inform. 2014, 10, 2364-2373. [CrossRef]

15. Lin, H.; Veda, S.S.; Shukla, S.S.; Mili, L.; Thorp, J. GECO: Global event-driven co-simulation framework for interconnected power system and communication network. IEEE Trans. Smart Grid 2012, 3, 1444-1456. [CrossRef]

16. Schütte, S.; Scherfke, S.; Tröschel, M. Mosaik: A framework for modular simulation of active components in Smart Grids. In Proceedings of the 2011 IEEE First International Workshop on Smart Grid Modeling and Simulation (SGMS), Brussels, Belgium, 17 October 2011; pp. 55-60.

17. Nguyen, V.H.; Besanger, Y.; Tran, Q.T.; Boudinnet, C.; Nguyen, T.L.; Brandl, R.; Strasser, T.I. Using power-hardware-in-the-loop experiments together with co-simulation for the holistic validation of cyber-physical energy systems. In Proceedings of the 2017 IEEE PES Innovative Smart Grid Technologies Conference Europe (ISGT-Europe), Torino, Italy, 26-29 September 2017; pp. 1-6. [CrossRef]

18. Awad, A.; Bazan, P.; German, R. SGsim: A simulation framework for smart grid applications. In Proceedings of the 2014 IEEE International Energy Conference (ENERGYCON), Cavtat, Croatia, 13-16 May 2014; pp. 730-736. [CrossRef]

19. Abdalkarim, A.; Bazan, P.; Reinhard, G. SGsim: Co-simulation Framework for ICT-Enabled Power Distribution Grids. In Measurement, Modelling and Evaluation of Dependable Computer and Communication Systems; Springer: Cham, Switzerland, 2016; pp. 5-8.

20. Bian, D.; Kuzlu, M.; Pipattanasomporn, M.; Rahman, S.; Wu, Y. Real-time co-simulation platform using OPAL-RT and OPNET for analyzing smart grid performance. In Proceedings of the 2015 IEEE Power Energy Society General Meeting, Denver, CO, USA, 26-30 July 2015; pp. 1-5. [CrossRef]

21. Venkataramanan, V.; Srivastava, A.; Hahn, A. Real-time co-simulation testbed for microgrid cyber-physical analysis. In Proceedings of the 2016 Workshop on Modeling and Simulation of Cyber-Physical Energy Systems (MSCPES), Vienna, Austria, 11 April 2016; pp. 1-6. [CrossRef]

22. Shum, C.; Lau, W.; Mao, T.; Chung, H.S.; Tsang, K.; Tse, N.C.; Lai, L.L. Co-Simulation of Distributed Smart Grid Software Using Direct-Execution Simulation. IEEE Access 2018, 6, 20531-20544. [CrossRef]

23. Primadianto, A.; Lu, C.N. A review on distribution system state estimation. IEEE Trans. Power Syst. 2017, 32, 3875-3883. [CrossRef] 
24. Ahmad, F.; Rasool, A.; Ozsoy, E.; Sekar, R.; Sabanovic, A.; Elitaş, M. Distribution system state estimation-A step towards smart grid. Renew. Sustain. Energy Rev. 2018, 81, 2659-2671. [CrossRef]

25. Cobelo, I.; Shafiu, A.; Jenkins, N.; Strbac, G. State estimation of networks with distributed generation. Eur. Trans. Electr. Power 2007, 17, 21-36. [CrossRef]

26. Haughton, D.A.; Heydt, G.T. A linear state estimation formulation for smart distribution systems. IEEE Trans. Power Syst. 2013, 28, 1187-1195. [CrossRef]

27. Pau, M.; Patti, E.; Barbierato, L.; Estebsari, A.; Pons, E.; Ponci, F.; Monti, A. Design and Accuracy Analysis of Multilevel State Estimation Based on Smart Metering Infrastructure. IEEE Trans. Instrum. Meas. 2019, 1-13. [CrossRef]

28. Patti, E.; Syrri, A.L.A.; Jahn, M.; Mancarella, P.; Acquaviva, A.; Macii, E. Distributed software infrastructure for general purpose services in smart grid. IEEE Trans. Smart Grid 2016, 7, 1156-1163. [CrossRef]

29. Estebsari, A.; Pons, E.; Patti, E.; Mengistu, M.; Bompard, E.; Bahmanyar, A.; Jamali, S. An iot realization in an interdepartmental real time simulation lab for distribution system control and management studies. In Proceedings of the 2016 IEEE 16th International Conference on Environment and Electrical Engineering (EEEIC), Florence, Italy, 7-10 June 2016; pp. 1-6.

30. Message Queue Telemetry Transport (MQTT). Available online: http:/ / mqtt.org/ (accessed on 4 April 2019).

31. InfluxDB. Available online: https://www.influxdata.com/ (accessed on 4 April 2019).

32. MongoDB. Available online: https://www.mongodb.com/ (accessed on 4 April 2019).

33. Bottaccioli, L.; Estebsari, A.; Pons, E.; Bompard, E.; Macii, E.; Patti, E.; Acquaviva, A. A flexible distributed infrastructure for real-time co-simulations in smart grids. IEEE Trans. Ind. Inform. 2017, 13, 3265-3274. [CrossRef]

34. Fielding, R.T.; Taylor, R.N. Architectural Styles and the Design of Network-Based Software Architectures; University of California: Irvine, CA, USA, 2000; Volume 7.

35. Eugster, P.T.; Felber, P.A.; Guerraoui, R.; Kermarrec, A.M. The many faces of publish/subscribe. ACM Comput. Surv. (CSUR) 2003, 35, 114-131. [CrossRef]

36. Patti, E.; Acquaviva, A.; Macii, E. Enable sensor networks interoperability in smart public spaces through a service oriented approach. In Proceedings of the 2013 5th IEEE International Workshop on Advances in Sensors and Interfaces (IWASI), Bari, Italy, 13-14 June 2013; pp. 2-7.

37. Mayer, C.P.; Gamer, T. Integrating Real World Applications into OMNeT++; Tech. Rep. TM-2008-2; Institute of Telematics, University of Karlsruhe: Karlsruhe, Germany, 2008.

38. Varga, A. Discrete event simulation system. In Proceedings of the European Simulation Multiconference (ESM'2001), Prague, Czech Republic, 6-9 June 2001.

39. Zhang, J.; Seet, B.C.; Lie, T.T. An event-based resource management framework for distributed decision-making in decentralized virtual power plants. Energies 2016, 9, 595. [CrossRef]

40. International Electrotechnical Commission (IEC). Available online: https://www.iec.ch/ (accessed on 4 April 2019).

41. Fernandes, C.; Borkar, S.; Gohil, J. Testing of goose protocol of IEC61850 standard in protection IED. Int. J. Comput. Appl. 2014, 93, 30-35. [CrossRef]

42. Amit, R.; Zott, C. Value creation in e-business. Strateg. Manag. J. 2001, 22, 493-520. [CrossRef]

43. Estebsari, A.; Patti, E.; Barbierato, L. Fault Detection, Isolation and Restoration Test Platform Based on Smart Grid Architecture Model Using Intenet-of-Things Approaches. In Proceedings of the 2018 IEEE International Conference on Environment and Electrical Engineering and 2018 IEEE Industrial and Commercial Power Systems Europe (EEEIC/I\&CPS Europe), Palermo, Italy, 12-15 June 2018; pp. 1-5.

44. Bletterie, B.; Kadam, S.; Renner, H. On the Classification of Low Voltage Feeders for Network Planning and Hosting Capacity Studies. Energies 2018, 11, 651. [CrossRef]

45. Presidential directive PDD-63. Available online: https://fas.org/irp/offdocs/pdd/pdd-63.htm (accessed on 4 April 2019).

46. Conrad, M.; Thomas, R. BDEW White Paper in practice: IT security in the secondary systems. In Proceedings of the International ETG-Congress 2013; Symposium 1: Security in Critical Infrastructures Today, Berlin, Germany, 5-6 November 2013; pp. 1-6.

47. ISO/IEC 27000 Family_Information Security Management Systems. Available online: https://www.iso. org/isoiec-27001-information-security.html (accessed on 4 April 2019). 
48. Bottaccioli, L.; Patti, E.; Macii, E.; Acquaviva, A. GIS-based Software Infrastructure to Model PV Generation in Fine-grained Spatio-temporal Domain. IEEE Syst. J. 2018, 12, 2832-2841. [CrossRef]

49. Weather Underground. Available online: http://www.wunderground.com (accessed on 4 April 2019).

50. Linke, F. Transmissions-koeffizient und Trübungsfaktor. Beitr. Phys. Fr. Atmos. 1922, 10, 91-103.

51. Šúri, M.; Hofierka, J. A new GIS-based solar radiation model and its application to photovoltaic assessments. Trans. GIS 2004, 8, 175-190. [CrossRef]

52. Erbs, D.; Klein, S.; Duffie, J. Estimation of the diffuse radiation fraction for hourly, daily and monthly-average global radiation. Sol. Energy 1982, 28, 293-302. [CrossRef]

53. Engerer, N. Minute resolution estimates of the diffuse fraction of global irradiance for Southeastern Australia. Sol. Energy 2015, 116, 215-237. [CrossRef]

54. Ruiz-Arias, J.; Alsamamra, H.; Tovar-Pescador, J.; Pozo-VÃ $j z q u e z$, D. Proposal of a regressive model for the hourly diffuse solar radiation under all sky conditions. Energy Convers. Manag. 2010, 51, 881-893. [CrossRef]

55. Karatasou, S.; Santamouris, M.; Geros, V. Analysis of experimental data on diffuse solar radiation in Athens, Greece, for building applications. Int. J. Sustain. Energy 2003, 23, 1-11. [CrossRef]

56. Reindl, D.T.; Beckman, W.A.; Duffie, J.A. Diffuse fraction correlations. Sol. Energy 1990, 45, 1-7. [CrossRef]

57. Skartveit, A.; Olseth, J.A. A model for the diffuse fraction of hourly global radiation. Sol. Energy 1987, 38, 271-274. [CrossRef]

58. Lin, W.M.; Teng, J.H.; Chen, S.J. A highly efficient algorithm in treating current measurements for the branch-current-based distribution state estimation. IEEE Trans. Power Deliv. 2001, 16, 433-439. [CrossRef]

59. Estebsari, A.; Pons, E.; Huang, T.; Bompard, E. Techno-economic impacts of automatic undervoltage load shedding under emergency. Electr. Power Syst. Res. 2016, 131, 168-177. [CrossRef]

(C) 2019 by the authors. Licensee MDPI, Basel, Switzerland. This article is an open access article distributed under the terms and conditions of the Creative Commons Attribution (CC BY) license (http:/ / creativecommons.org/licenses/by/4.0/). 